\title{
Kangxi/Engke Amuyulang, un empereur mongol ? Sur quelques légendes mongoles et chinoises
}

Kangxi/Engke Amuyulang, a Mongolian Emperor? On some Mongolian and Chinese stories

Isabelle Charleux

\section{OpenEdition}

Journals

Édition électronique

URL : https://journals.openedition.org/emscat/1782

DOI : 10.4000/emscat.1782

ISSN : 2101-0013

Éditeur

Centre d'Etudes Mongoles \& Sibériennes / École Pratique des Hautes Études

Référence électronique

Isabelle Charleux, «Kangxi/Engke Amuyulang, un empereur mongol ? Sur quelques légendes

mongoles et chinoises », Études mongoles et sibériennes, centrasiatiques et tibétaines [En ligne], 42 |

2011, mis en ligne le 20 décembre 2011, consulté le 13 juillet 2021. URL : http://

journals.openedition.org/emscat/1782 ; DOI : https://doi.org/10.4000/emscat.1782 


\title{
Kangxi/Engke Amurulang, un empereur mongol ? Sur quelques légendes mongoles et chinoises
}

\author{
Isabelle Charleux (CNRS - GSRL)
}

La période dite « de la domination mandchoue » (dynastie Qing (1636/1644 ${ }^{1}$-1911), marquée par la perte de l'autonomie, la colonisation et l'exploitation des Mongols, ${ }^{2}$ est depuis un siècle une période mal aimée, en particulier des Mongols de Mongolie, qui considèrent les Qing comme responsables de nombreux maux de la Mongolie moderne ${ }^{3}$.

Pourtant, le règne de Kangxi 康熙 (r. 1662-1722) ${ }^{4}$ se distingue par son image positive : les nombreuses histoires qui circulaient et circulent encore sur le deuxième empereur de cette dynastie montrent que les Mongols, loin de le présenter en despote étranger opprimant son peuple, le considéraient comme un souverain parfaitement légitime, voire même, comme un empereur mongol. C'est d'ailleurs le seul souverain mandchou qui fait l'objet de légendes en Mongolie.

Comment peut-on expliquer que des récits véhiculent une image positive de Kangxi alors que l'ensemble de la période Qing a aujourd'hui une image négative ? Ces histoires véhiculent-elles une image de l'empereur qui serait antérieure à la chute de l'empire ? Ontelles été propagées délibérément par le pouvoir mandchou? Trouve-t-on des récits plus positifs chez les Qorcin, groupe auquel appartenait la grand-mère de Kangxi, et des histoires plus critiques vis-à-vis de son règne chez les Qalqa et les Tümed ? Il importe de comprendre d'où viennent ces histoires, comment et dans quel milieu elles ont circulé, et quelle est la responsabilité du pouvoir impérial dans la transmission d'images positives de Kangxi. Je mettrai en parallèle, lorsque cela est possible, l'image qu'il donnait de son vivant et le Kangxi légendaire, afin d'évaluer dans quelle mesure les bases historiques de ces légendes - lorsqu'elles existent - ont été transformées et déformées par les traditions orales.

Je propose ici de présenter quelques récits oraux provenant des Qorcin, des Tümed et des Ordos de Mongolie-Intérieure, ainsi que des Qalqa de Mongolie, qui ont été publiés dans des recueils de contes et des monographies locales. Ces histoires ont été collectées par

\footnotetext{
${ }^{1}$ La dynastie Qing est proclamée en 1636 lorsque la plupart des Mongols méridionaux (résidant dans ce qui allait devenir la Mongolie-Intérieure) se rallient; elle renverse la dynastie Ming en 1644.

${ }^{2}$ Par «mongol», sans autre spécification, je ne ferai ici référence qu'aux Mongols septentrionaux et méridionaux habitant aujourd'hui respectivement en République de Mongolie (ancienne Mongolie-Extérieure) et en Mongolie-Intérieure, et dont l'aristocratie descendait de Chinggis Khan et de sa famille. Une grande partie des Mongols occidentaux ayant été exterminée par les Qing, ceux-ci ont pour la plupart bien plus de raisons de haïr Kangxi que de l'aimer.

${ }^{3}$ Cependant, des publications récentes de chercheurs occidentaux comme Christopher Atwood et Johan Elverskog ainsi que l'essor des études en (République de) Mongolie et en Mongolie-Intérieure (province chinoise) tentent depuis peu de «réhabiliter » la dynastie Qing d'un point de vue mongol et de souligner l'indéfectible loyauté des Gengiskhanides envers la maison impériale mandchoue.

${ }^{4}$ Ma. Elhe taifin, mong. Engke Amurulang, 1654-1722. Kangxi est son nom de règne ; son nom personnel était Xuanye 玄燁.
} 
des ethnographes et des folkloristes dans la deuxième moitié du $\mathrm{Xx}^{\mathrm{e}}$ siècle, auprès de conteurs mongols, et sont encore répandues chez les Qorcin. Les recueils de littérature orale ne fournissent généralement pas de précisions sur le contexte et l'origine de ces contes, ni sur l'éventuelle mélodie qui les accompagne ${ }^{5}$. Un chercheur de MongolieIntérieure, B. Uranchimeg, m'a rapporté trois histoires sur Kangxi voyageant incognito sur son âne, contées dans sa famille qorcin ${ }^{6}$. J'ai limité mon étude aux histoires dont Kangxi est le héros ; mais l'empereur apparaît encore dans d'autres légendes mongoles comme un personnage secondaire, en particulier dans les anecdotes sur le troisième Mergen gegeen Lubsangdambijalsan (1717-1766) ${ }^{7}$.J'ai recensé des lieux où l'on commémore le passage de l'empereur ainsi que des représentations picturales de ces légendes. La comparaison avec quelques histoires chinoises qui circulent au Mont Wutai 五臺山 (province du Shanxi en Chine), lieu de pèlerinage populaire chez les Mongols au XIX ${ }^{\mathrm{e}}$ et au début $\mathrm{du} \mathrm{XX}^{\mathrm{e}}$ siècle (Charleux, à paraître), permettra enfin d'introduire quelques hypothèses sur les possibles modes de transmission du discours mongol sur Kangxi.

\section{Pourquoi spécifiquement Kangxi ?}

$\mathrm{Au}$ préalable, pourquoi tant d'histoires portent sur l'empereur Kangxi (Engke

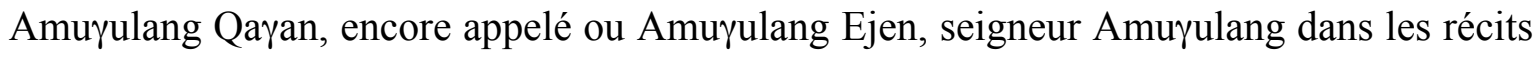
mongols) plutôt que, par exemple, sur ses prédécesseurs Hungtaiji (r. 1627-1644) ou Qianlong (r. 1736-1795)? Tout d'abord, Kangxi avait du sang mongol. Ses parents moururent tôt, et le jeune prince fut élevé par sa grand-mère, l'impératrice douairière Xiaozhuang 孝莊 (1613-1688), une Mongole qorcin descendante de Joci (encore appelé Qabutu Qasar, un frère de Chinggis Khan). Kangxi conserva toute sa vie une affection sans bornes pour sa grand-mère. Respectée pour sa sagesse et son habileté politique, Xiaozhuang exerça une grande influence sur le jeune empereur et sur la cour : ainsi Kangxi recueillit par écrit les mots et préceptes de Xiaozhuang pour les transmettre à ses propres enfants (Hummel 1943-1944, pp. 300-301).

En second lieu, menant une politique de conquêtes, Kangxi se retrouva entraîné dans des conflits intra-mongols. Rappelons que depuis 1636, les Mongols méridionaux reconnaissaient l'empereur mandchou comme leur souverain, héritier de Chinggis Khan et de Khubilai Khan, alors qu'au début du règne de Kangxi, les Mongols qalqa formaient encore des royaumes indépendants. En 1688, les Qalqa, agressés par les Züüngar ${ }^{8}$, demandèrent l'aide de l'empire Qing. Kangxi s'investit personnellement dans le conflit malgré l'opposition de ses ministres, et mena en 1690 et 1696 deux campagnes contre les Züüngar. Les armées Qing furent deux fois victorieuses, mais subirent de très lourdes

\footnotetext{
${ }^{5}$ La littérature orale - des épopées chantées par les bardes aux fables accompagnées à la viole, chants chamaniques et proverbes - est abondante et variée dans le monde mongol. Les folkloristes et ethnographes mongols de Mongolie et de Mongolie-Intérieure (pour ces derniers, voir l'avant-propos in Chiodo 2011), ainsi que des mongolisants occidentaux comme Walther Heissig, ont publié plusieurs collections sur la littérature orale.

${ }^{6}$ Ces histoires lui ont été contées par son père, Bao Jinshan, né dans l'aile du milieu de la bannière orientale des Qorcin en 1911.

${ }^{7}$ Heissig 1999, pp. 81-82 ; Humphrey 2010 ; pp. 202-203.

${ }^{8}$ Mongols occidentaux qui nomadisaient à l'ouest de l'empire Qing.
} 
pertes $^{9}$. En 1690, Kangxi rétablit les Qalqa sur leur territoire mais dut porter secours aux 200000 Mongols qui s'étaient réfugiés dans l'empire et les sauver de la famine ${ }^{10}$. C'est grâce à cette première victoire qu'en 1691 les Qalqa firent allégeance à l'empire Qing lors de la Convention de Dolonnor. Les guerres züüngar sont à resituer dans le contexte de la politique coloniale de l'empire mandchou, mais, du point de vue des Qalqa, Kangxi déploya d'impressionnants moyens pour venir à leur secours. Ces guerres coûtèrent en effet cher à l'empire, en finances et en hommes.

Kangxi était aussi connu des Mongols comme l'auteur des "Saintes instructions » (Shengyu guangxun 聖諭廣訓, publié en 1724), texte d'instruction morale en 16 maximes, qui fut augmenté par Yongzheng (Shengyu guangxun 聖諭廣訓) - cette deuxième version fut traduite en mongol et très utilisée comme manuel élémentaire pour les écoliers chinois et mongols. Atwood (2000, pp. 115-116) a montré que ce texte a été un des véhicules pour la transmission de la « rhétorique de la grâce » impériale auprès des Mongols.

Enfin Kangxi ne s'est pas seulement proclamé empereur des Mongols ; il s'est aussi comporté comme un empereur mongol. C'est sans doute ce que ressentait le vieux guerrier Caqundorzi, prince Tüsijetü qan des Qalqa, qui pleura d'émotion lorsqu'il dit au revoir à l'empereur à l'issue de la Convention de Dolonnor en $1691^{11}$. À la fois guerrier, chasseur, pieux bouddhiste et souverain généreux, Kangxi s'est assuré la complète loyauté de ses sujets mongols. Sa politique mongole a été couronnée de succès, puisque les Mongols intégrés à l'empire ont pleinement eu le sentiment d'appartenir à «nos grands Qing » comme l'a si bien montré J. Elverskog (2006). Cette loyauté revenait à l'empereur et non à l'empire et à ses institutions: "The K'ang-hsi emperor obviously possessed this immediate personal appeal, the necessary charisma and 'fortune,' without which no Central Asiatic leader could be acceptable » (Veit 1982, p. 350). Ce n'est pas à l'empire Qing que les Mongols faisaient allégeance, mais à la personne de l'empereur.

\section{Kangxi, le digne héritier de Chinggis Khan ?}

\section{Un empereur juste qui sait pardonner}

Dans plusieurs histoires mongoles, Kangxi se montre magnanime et juste. Deux anecdotes concernant la fondation de monastères mettent en scène des nobles qui ont commis une faute mais se font ensuite pardonner. Rappelons que les princes mongols de l'empire épousaient fréquemment des princesses impériales mandchoues et devenaient ainsi «gendres impériaux ». Devenus «parents » de l'empereur, certains outrepassaient leurs privilèges.

- Au XVIII ${ }^{\mathrm{e}}$ siècle, le duc Galsang (1692-1711) de la bannière de droite des Qaracin se fit construire un palais bien trop luxueux pour son rang. Aussi, lors d'une tournée

\footnotetext{
${ }^{9}$ Les Züüngar continuèrent toutefois de menacer l'empire et ne seront vaincus qu'en 1757 par Qianlong.

${ }^{10}$ Perdue considère le ravitaillement des réfugiés en nourriture comme «a useful weapon in the conquest of Mongolia » (2005, p. 177).

${ }^{11}$ Veit 1982, p. 350, citant le Iledkel šastir (Tableaux [généalogiques] et biographies des ducs et des princes des tribus mongoles et turques, 1779-1789) 46, 12r.
} 
d'inspection de l'empereur Kangxi, s'empressa-t-il de le doter d'une statue du bouddha Shākyamuni afin de le transformer en temple. Heureux d'avoir ainsi préservé son palais, il le nomma Lingyuesi 靈悅寺 (《Monastère du bonheur numineux ») (Qiao Ji 1994, p. 97).

- Le Darqan vang des Qorcin, oncle maternel de Kangxi, avait aidé ce dernier à monter sur le trône pendant la cérémonie d'intronisation. Lorsqu'il se rendait à Pékin, l'empereur reconnaissant venait l'accueillir en personne hors de la ville. Mais des subalternes médirent de ces honneurs rendus à un prince mongol et Kangxi cessa de lui manifester ce privilège. Le Darqan vang, furieux, fit alors le serment de se rebeller contre les Qing. Kangxi délégua un haut dignitaire religieux, le Caץan dijanci quturtu des Tümed, qui parvint à apaiser le prince. Pour conjurer le serment, le grand lama convainquit le Darqan

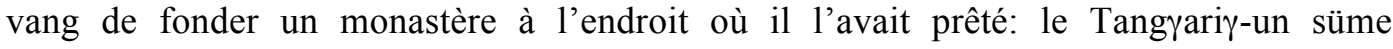
(« monastère du serment ») (Delege 1998, p. 681).

Kangxi avait la réputation d'être un empereur juste : à l'instar de Chinggis Khan, il récompensait la loyauté et la bravoure par l'octroi de grâces impériales, mais punissait sévèrement la couardise, la désobéissance et par-dessus tout, la trahison (Spence

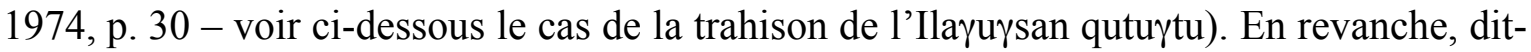
on, il pardonnait facilement les petites fautes sans se formaliser : alors que ses fils et gardes du corps s'apprêtaient à battre le cuisinier qui apportait des plats de viande sans riz, l'empereur les en empêcha: «It wasn't intentional, they just forgot to bring it : it's a small thing, we can let it pass » (Spence 1974 p. 17).

\section{Un empereur (trop) magnanime}

Dans une histoire de la province Dundgov' en République de Mongolie, qui rappelle les contes de la bru censée ${ }^{12}$ mais aussi les recueils de fables indiennes comme le Pañcatantra ${ }^{13}$, l'empereur excessivement généreux est pris au piège de sa réputation, et c'est grâce à un enfant de huit ans qu'il ne perd pas la face :

L'empereur Kangxi était réputé être à tel point magnanime que dans son pays, il n'y avait ni mendiant ni chien errant. Il avait fait le serment d'exaucer les vœux de tous et ne jamais dire «non ». Trois hommes mal intentionnés d'un pays voisin décidèrent de le mettre à l'épreuve et de lui faire dire "non ». Ils demandèrent à Kangxi trois choses impossibles : de la soie pour couvrir le monde entier, de la farine pour remplir la Mer Extérieure jusqu'à ce qu'elle sèche, et de l'or pour recouvrir tout le mont Sumeru. Kangxi, lié par son serment, leur promit de donner ce qu'ils demandaient. Il rassembla les sages de tout son pays pour trouver une solution. L'empereur entendit alors une conversation au sujet d'un de ses ministres dont le fils de huit ans parlait de manière compliquée et énigmatique. L'enfant dit à son père que les ministres se tracassaient pour des petites choses en croyant qu'elles étaient de grands problèmes. Il incita son père à demander à l'empereur une audience privée et à lui répéter ce qu'il allait lui dire afin de se débarrasser définitivement des trois hommes mal intentionnés. Il proposa de donner l'ordre aux trois hommes de partir prendre les mesures du monde, du Sumeru et de la Mer Extérieure, afin d'évaluer quelles quantités exactes de soie, d'or et de farine étaient nécessaires pour satisfaire leurs vœux : alors seulement l'empereur accèderait à leur demande. On dit qu'ils n'ont toujours pas fini de prendre les mesures, et qu'ils sont devenus respectivement têtard, ver et chenille (Cerensodnom 1982, pp. 193-195).

\footnotetext{
${ }^{12}$ Voir Hamayon et Bassanoff 1973.

${ }^{13}$ Cette histoire pourrait provenir d'Inde via des textes bouddhiques, mais je n'en ai pas trouvé l'origine.
} 


\section{Un empereur bouddhiste}

Dans le grand rituel cam (< tib. 'cham) d'Ourga, la figure de Hašin ${ }^{14}$ han [*Qasin qan], le "roi-moine ", accompagné de ses huit fils, est souvent identifiée à l'empereur Kangxi, car ce dernier a grandement contribué à la propagation du bouddhisme en Mongolie (fig. 1). Hašin han apparaît comme la figure du donateur exemplaire dont le rôle est de soutenir financièrement et de protéger l'Église bouddhique. Lors du rituel, Hašin han et ses fils forment une sorte de comité de réception des danseurs, au cou desquels ils nouent une écharpe cérémonielle. Il porte un masque jaune de dimensions disproportionnées et une robe jaune, et fait à plusieurs reprises le tour de l'assistance avec ses enfants en lançant des bonbons (Majer 2008, p. 109) ${ }^{15}$. En fait, Hašin/Hwa-shang est dans le panthéon tibétomongol le dix-septième arhat, Hwa-shang, moine chinois de l'école de méditation (Chan) qui aurait participé au Concile de Lhasa en 792. Il est amalgamé à la figure de Budai heshang 布袋和尚 (le moine au sac de toile), ventru et hilare, lui-même identifié à Maitreya et accompagné d'enfants ${ }^{16}$. Les Qalqa en ont fait une figure royale en lui adjoignant le titre de han (khan) et l'ont identifié à Kangxi. Il est intéressant de noter que dans le cam, ce personnage est tantôt vénérable, tantôt ridicule.

L'empereur Kangxi se présentait effectivement aux yeux des Mongols comme un empereur bouddhiste. De même que ses prédécesseurs, il était considéré comme émanation du bodhisattva Mañjuśrī et souverain qui fait «tourner la roue de la Loi » pour propager les enseignements bouddhiques (Farquhar 1978). Les chroniques mongoles le désignaient sous l'appellation de « Saint empereur Mañjuśrī ${ }^{17}$. Kangxi montra un intérêt marqué pour le bouddhisme tibétain ; ils entretint entre autres des relations privilégiées avec Zanabazar, premier de la lignée des Jebcündamba, qui fut invité à la cour de Pékin où il accomplit des miracles, si l'on en croit sa biographie (fig. 2) ${ }^{18}$. Toutefois, son soutien au bouddhisme n'apparaît généralement pas dans les histoires mongoles.

\section{Un empereur généreux}

Fidèle à sa formule « construire un seul temple équivaut à nourrir cent mille soldats en Mongolie », Kangxi fit édifier des monastères fastueux pour les Mongols, en MongolieIntérieure et à Chengde. D'autres monastères sont dits avoir été fondés à l'occasion des chasses de Kangxi, pour commémorer une halte, ou sur ordre de l'empereur lui-même.

Kangxi se montra particulièrement généreux envers les monastères de Hohhot (mong. Kökeqota), car la ville et la région alentour, ayant servi de base militaire à la

\footnotetext{
${ }^{14}$ Encore écrit en cyrillique Huušan ou Huušin, < tib. hwa-shang, < ch. heshang 和尚, moine (chinois).

${ }^{15}$ On le trouve encore identifié chez les Qalqa à un roi du Cachemire («Face Music» 2007), à un prince mongol qui aurait invité un saint indien pour soigner l'empereur des Ming (Fontein 1999, p. 50); ou encore au roi mongol Abatai qan, fondateur du monastère Erdeni zuu (Pegg 2001, pp. 160-161).

${ }^{16} \mathrm{Ce}$ débat religieux (qui eut lieu en réalité au monastère de Samyé) opposa les tenants du bouddhisme indien et du bouddhisme chinois; les premiers l'emportèrent. Pour les Tibétains, Hwa-shang représente l'archétype du moine chinois, mais aussi, à la frontière chinoise, une figure politique liée à l'autorité chinoise (Schrempf 2006). Sur son origine : Schrempf 2006, pp. 4-5.

${ }^{17}$ Références dans Atwood 2000.

${ }^{18}$ Bawden (éd.) 1961,p. 60, citant une biographie du Jebcündamba qutuүtu.
} 
conquête du Turkestan, avaient contribué à l'effort de guerre. Hohhot dut stocker de grandes quantités de grains pour nourrir les armées mandchoues et les réfugiés qalqa auxquels l'empereur accorda asile lors de ses deux campagnes contre les Züüngar. De plus, les Mongols avaient fourni un grand nombre de soldats non seulement dans les guerres züüngar, mais aussi pour mater la rébellion des Trois feudataires (1673-1681) et les révoltes dans le sud de la Chine.

Lorsque l'empereur séjourna à Hohhot en 1696-1697, il résida au Siregetü zuu et au Baya zuu ("Petit monastère»), siège du $\mathrm{II}^{\mathrm{e}}$ Neici toin quturtu, et favorisa tout particulièrement ce dernier monastère en raison de l'estime qu'il avait pour son abbé. Il en finança l'agrandissement et offrit des objets personnels et des cadeaux somptueux : des sutras, des robes monastiques, « un coussin de trône brodé en fils d'or, un dossier brodé d'or, un oreiller brodé d'or, deux arcs précieux, des flèches grandes et petites, cinq armures complètes, des peaux de tigre et de léopard. On les exposait chaque année, le $12 \mathrm{du} \mathrm{VI}$ mois » (Guisui xian zhi [1934] 1968, p. 303). Selon la stèle de 1703 qu'il fit dresser pour glorifier en quatre langues la victoire mandchoue, il offrit encore : « un casque et une cotte de maille que j'ai [qu'il a] personnellement portés, un arc et des flèches que j'ai [qu'il a] personnellement utilisés, un arc et une flèche que je gardais [qu'il gardait] avec moi [lui] ; et un carquois, ainsi qu'un étui à arc que je [qu'il] gardait attachés $\gg{ }^{19}$.

Plusieurs monastères de Mongolie orientale conservaient ou prétendaient conserver des objets ayant appartenu à Kangxi. Retournant à Shenyang après la victoire contre Galdan, chef des Züüngar, Kangxi s'arrêta au Longquansi 龍泉寺, vint y brûler de l'encens en 1697 ou 1698, et offrit arc, flèches et bride en jade (Charleux 2006, [112]). Le monastère du Cayan dijanci de Fuxin 阜新 (situé dans la province du Liaoning) préservait une bride ayant, dit-on, appartenu à l'empereur. On rapporte encore que Kangxi, voyageant incognito à dos d'âne, aurait passé la nuit dans le petit temple du village mongol de Bainiucun ${ }^{20}$, perché sur un massif escarpé, et laissé - volontairement ou par oubli - une botte et des bas, qui constituaient au début du siècle un trésor inestimable. Les reliques étaient exposées une fois par an lors du festival du temple, et des milliers de Mongols pieux venaient offrir de l'encens et se prosterner en les touchant de leur tête (Hedley 1910, p. 96).

Ces objets personnels étaient des trésors précieusement conservés par les monastères : Kangxi était non seulement le «Saint Empereur Mañjuśrī» - un empereur-dieu qui transformait tout ce qu'il touchait en relique sacrée -, mais également, à l'instar des grands Khan charismatiques, il était considéré comme une divinité protectrice des Mongols. Ses armes dotées des pouvoirs surnaturels de leur ancien propriétaire assuraient symboliquement la protection du monastère ${ }^{21}$.

\footnotetext{
${ }^{19}$ Guisui xian zhi 1934, p. 454 ; version mongole traduite par Pozdneev [1896-1898] 1977, p. 40.

${ }^{20}$ Dans la bannière du centre des Qaracin (dans l'actuel Liaoning), à une centaine de kilomètres à l'est de Chengde.

${ }^{21}$ Kangxi offrit également au temple du Roi Dragon (Wuyemiao 五岳廟) du Wutaishan des armes réputées particulièrement efficaces contre les démons.
} 


\section{Kangxi divinisé}

Kangxi apparaît ainsi aux yeux des Mongols comme le "saint empereur » sage, bienveillant et tout puissant qui sacralise les lieux qu'il visite, dont l'archétype pour les Mongols est, naturellement, Chinggis Khan (Birtalan 2005). Un chant mongol d'époque Qing loue «Notre Saint Souverain, l'Empereur, incarnation de Mañjuśrī, [qui] aime profondément ses sujets mongols et mandchous » (cité par Atwood 2000, p. 88 - notons que pour les Mongols, les Chinois ne sont pas inclus dans cette relation). Il chérissait son peuple et octroyait ses «grâces » (kesig) sous forme de cadeaux, de récompenses (titres, salaires...) et de reliques ${ }^{22}$; or chez les peuples des steppes, le chef se doit de distribuer à chacun sa part (de butin, de cadeaux) afin d'augmenter son prestige et de légitimer son autorité.

Parce qu'il avait écrasé l'armée de Galdan et sauvé les Qalqa de la famine, Kangxi a été considéré par ces derniers comme «un khan aux pouvoirs magiques » (Perdue 2005, p. 190). Comme Chinggis Khan, il a également été crédité de quelques actes miraculeux, preuves de sa légitimité à régner et de son origine « divine ». À $100 \mathrm{~m}$ au sud de l'entrée du principal monastère de Hohhot, le Jeke zuu (« Grand monastère », ch. Dazhao 大召) se trouvait la 《source impériale» (Yuquan 御泉), par la suite appelée par homonymie 《source de jade»(Yuquan 玉泉) en raison de la pureté de son eau, avec deux mâts, une source, huit petits puits, et une stèle (fig. 3). Cette source aurait jailli, dit-on, sous le pied du cheval de Kangxi revenant, assoiffé, de la guerre contre les Züüngar en $1696^{23}$ (fig. 4). La source sacrée, devenue par la suite neuf puits, était l'objet d'un culte populaire, et un temple lui fut dédié, le Yuquanmiao 玉泉廟. L'eau était donnée en particulier aux parturientes ainsi qu'aux gens ivres pour les faire dessoûler. Bien que la source se fût tarie à la suite d'un tremblement de terre, elle a continué à être vénérée jusqu'à la fin du $\mathrm{XX}^{\mathrm{e}}$ siècle : en 1997 une petite enceinte a été bâtie autour des puits, avec une peinture illustrant le passage de Kangxi et un bas-relief du dieu dragon. L'ensemble a été rasé lors de la modification du quartier au début des années 2000 .

Un autre exemple est le Batu qayalya-yin süme ${ }^{24}$, un des principaux monastères de l'ouest de la Mongolie-Intérieure, qui aurait été fondé de 1702 à 1705 sur un site où se serait arrêté Kangxi pendant sa deuxième campagne contre Galdan. Au sud-ouest du monastère, un lieu est nommé «cuvette du campement de Kangxi » (Kangxi yingpan 營 盤); au sommet de la montagne, une pierre porte l'empreinte du sabot du cheval de Kangxi - qui en réalité n'est jamais venu à cet endroit (Lu Minghui 1987, p. 164). Seuls

\footnotetext{
${ }^{22}$ Les faveurs impériales sont par définition disproportionnées par rapport aux mérites du récipiendaire, de sorte que ce dernier a toujours le sentiment qu'il ne les mérite pas et, craignant de ne jamais pouvoir remercier, se retrouve ainsi fortement endetté : voir Atwood 2000.

${ }^{23}$ L'anecdote est rapportée dans la monographie officielle Guisui xian zhi. Voir Zhao Chunping et al. 1987, pp. 50-51. On retrouve une légende similaire en Hongrie, où le roi Ladislaus ${ }^{\mathrm{er}}$, poursuivi par les Comans, aurait sauté avec son cheval dans un précipice et atterri sur un sol meuble. Du sabot de son cheval jaillit une source sacrée. Cette légende explique l'origine de l'eau miraculeuse de Notre-Dame de Matraverebely (Szentkút), principal site de pèlerinage marial en Hongrie (communication de Saskia Kersenboom au colloque «Pilgrimages and Sanctuaries: Art, Music and Rituals », Ascona, 11 novembre 2011).

${ }^{24}$ Encore appelé Beile-jin süme, ch. Bailingmiao 百靈廟, dans la municipalité de Boyutu/Baotou (Charleux $2006,[74])$.
} 
les saints, les bouddhas et Chinggis Khan laissent habituellement de telles marques sur le paysage. Parmi les autres lieux sacralisés par le passage de Kangxi, on trouve encore le

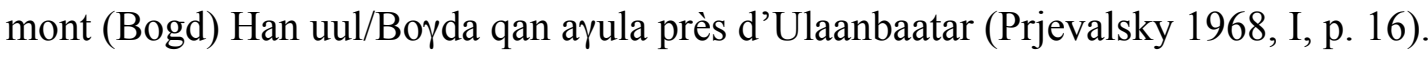

\section{Kangxi, l'empereur chasseur}

Tels les anciens souverains d'Asie intérieure, les empereurs mandchous pratiquaient la chasse comme un rituel royal (Allsen 2006). Kangxi, quant à lui, avait une véritable passion pour cette activité. Il chassait, à l'arc ou au fusil, lors de «petites chasses » (aux cerfs, lièvres, faisans) et de " grandes » (aux tigres), et ses trophées les plus célèbres furent des tigres. Sa biographie montre une obsession du décompte (135 tigres, 20 ours, etc. $)^{25}$. L'empereur chassait pendant tous ses déplacements en Mongolie ${ }^{26}$. Les voyages aux tombes de ses ancêtres à Mukden étaient souvent prétextes à des excursions de chasse chez

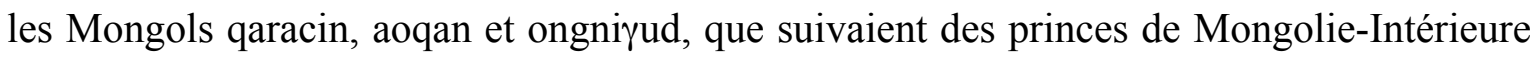
et deux mille cavaliers mongols, ainsi que quelques soldats mandchous et des officiers de Pékin.

Spence (1974, p. xv et chap. 1) montre d'ailleurs que la seule guerre que Kangxi mena en personne - la campagne de 1696-1697 - s'apparenta plus à une chasse pour obtenir le trophée de la tête de Galdan qu'à une campagne militaire ${ }^{27}$. L'empereur reçut la nouvelle $\mathrm{du}$ suicide de son ennemi comme un triomphe personnel. À l'inverse, ses chasses mongoles ressemblaient souvent plus à une expédition militaire qu'à une partie de divertissement : c'étaient aussi des démonstrations de la puissance militaire mandchoue, rassemblant parfois jusqu'à 60000 hommes. En 1681, au retour d'une de ses expéditions de chasse, les Qaracin lui offrirent les terres de Mulan 木兰 (à 120 kilomètres au nord de Chengde) comme réserve de chasse, et c'est sous son règne que la chasse impériale devint une cérémonie institutionnalisée accompagnée de banquets et de festivités variées. Selon Wei Yuan (Shengwu ji, chap. 3, 9a), la chasse était le meilleur rituel pour s'assurer la fidélité des Mongols. Les chasses étaient à la fois un partage d'expériences communes l'empereur impressionnait les princes mongols par sa bravoure (Ripa 1844, p. 77) et montrait qu'il était culturellement proche d'eux - et une démonstration de supériorité ${ }^{28}$. Des relations personnelles pouvaient ainsi se former entre l'empereur et les princes mongols lors des chasses et des rituels de Nouvel An. Sous ses successeurs, les relations entre Mandchous et Mongols devinrent beaucoup plus formelles.

Les exploits de chasse de Kangxi ont marqué les Mongols, et plusieurs monastères possèdent des peaux de tigres prétendument tués par l'empereur. Ainsi le Huifusi 薈福寺

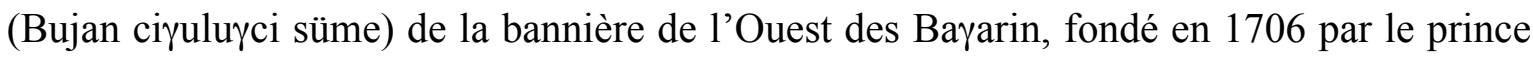

\footnotetext{
${ }^{25}$ Spence 1974, chap. 1, en particulier p. 9. J. Spence est l'auteur d'une biographie de Kangxi fondée sur des écrits personnels de l'empereur (lettres à ses proches, conversations avec ses fils, essais, mémoires du palais). ${ }^{26}$ Comme le constata le père Gerbillon (Du Halde 1735, IV pp. 77, 128, 260-261, 280-281, 290-301, 346).

${ }^{27}$ Kangxi avait d'abord cherché à pacifier les deux parties, mais qu'après 1690 , sous prétexte de défendre les Qalqa, il mena une campagne personnelle contre les Züüngar jusqu'à la mort de leur chef (Perdue 2005, chapitres 4 et 5). L'historiographie Qing fit par la suite de Galdan un envahisseur et un criminel.

${ }^{28}$ De même, l'expédition de Kangxi en Mongolie-Intérieure en 1696 était un déploiement de richesses et de puissance militaire visant à impressionner les Mongols (Perdue 2005, p. 195-196).
} 
Ürgün, marié à la princesse mandchoue Rongxian 榮憲 (troisième fille de Kangxi), est surnommé Bars süme, « temple du tigre », car on dit que Kangxi qui venait chasser le tigre dans la région y avait suspendu une peau de tigre (Charleux 2006, [128]).

Certains de ces récits sont issus de faits réels, ainsi les Mongols qui se rendaient en pèlerinage au Wutaishan pouvaient entendre celui de la fameuse chasse de Kangxi (fig. $\mathbf{5}^{29}$. Lors de sa première visite au Wutaishan en 1683 , alors qu'il quittait la montagne, l'empereur surprit un tigre à moitié caché dans des buissons sur la route de Changchengling 長城嶺 (porte d'une portion de grande muraille sur la route WutaishanPékin); le tigre s'enfuit en grimpant la montagne, l'empereur le poursuivit et le tua d'une flèche. Pour commémorer l'événement, le site fut renommé Shehuchuan 射虎川, «Vallée où le tigre fut tué d'une flèche» et un monastère y fut édifié, le Tailusi 台麓寺. On y préservait la peau du tigre tué par Kangxi, ou selon d'autres sources, une (statue de) tigre qui ressemblait à un vrai. Kangxi fit ensuite ériger à Shehuchuan une stèle commémorant son exploit ${ }^{30}$, dont le texte figure au début de la monographie locale impériale Qinding Qingliangshan zhi 欽定清凉山志 (chap. 1, 6). L'histoire apparaît encore au début du chapitre sur les «Sites célèbres » de cette même monographie ${ }^{31}:$ l'épisode a donc pris des proportions importantes dans l'histoire impériale. Kangxi n'est plus seulement le viril roi chasseur d'Asie intérieure et le parfait guerrier mandchou qui protège le petit peuple en tuant des fauves ${ }^{32}$ : il s'inscrit également dans la lignée d'anciens empereurs chasseurs au Wutaishan, dont il « rejoue » les exploits civilisateurs ${ }^{33}$. Enfin, en débarrassant la région de ce prédateur, il reproduit la subjugation par Mañjuśrī des cinq cent dragons qui s'opposaient à l'implantation du bouddhisme ${ }^{34}$. C'est à travers ces histoires, ainsi qu'à travers les stèles et la réécriture des monographies locales que Kangxi inscrit son mythe personnel dans l'histoire des différentes régions de son empire ${ }^{35}$.

La carte du Wutaishan gravée en 1846 au Cifusi 慈福寺 par un lama mongol, et destinée en particulier aux pèlerins mongols, illustre cet épisode qui avait pris de nouvelles proportions légendaires plus de cent ans après les visites de l'empereur ${ }^{36}$. Des pèlerins mongols à qui je montrais cette carte eurent la réaction suivante : certes ce fauve menaçait des pèlerins, mais comment peut-on tuer un animal sur une montagne sacrée, terre pure du

\footnotetext{
${ }^{29}$ Kangxi s'est rendu à cinq reprises au Wutaishan. À l'exception de sa visite avec sa grand-mère en 1683 , ces voyages n'étaient pas des pèlerinages mais des tours d'inspection ayant un but essentiellement politique et culturel (Köhle 2008, pp. 91-98).

30 《Shehuchuan Tailusi bei »射虎川台麓寺碑, et 《Tailusi bei »臺麓寺碑 (Zhang Yuxin 1988, pp. 242243, 259-260).

${ }^{31}$ Voir Köhle 2008, p. 93 ; Chou 2007, p. 123.

${ }^{32}$ On pense à l'histoire célèbre de Wu Song 武松, personnage du roman chinois Au bord de l'eau (traduit en mongol au XIX ${ }^{\mathrm{e}}$ siècle), qui tua à mains nues un tigre qui terrorisait la population.

${ }^{33}$ D'après la stèle mentionnée ci-dessus (Chou 2007, p. 124). Les histoires d'empereurs chasseurs commémorant leurs exploits par des stèles sont des lieux communs de l'histoire chinoise. Le Wutaishan était d'ailleurs une réserve de chasse impériale au V' siècle (Chou 2007, p. 123).

${ }^{34}$ De même dans les danses rituelles masquées cam en Mongolie, le Vieillard Blanc tue un tigre-démon.

${ }^{35}$ Köhle 2008,pp. 99.

${ }^{36}$ On connaît au moins une douzaine d'exemplaires de cette carte qui a circulé dans le monde mongol (Halén 1987 ; Chou 2007).
} 
bodhisattva Mañjuśrī $?^{37}$ C'est certainement pour cette raison que la légende chinoise suivante rejette la faute sur les gardes de l'empereur et offre une « réparation » :

Kangxi en pèlerinage au Wutaishan logea au palais de voyage du Tailusi. Au crépuscule, il vit un tigre descendre la montagne et demanda à ses gardes : " Les tigres mangent-il les hommes? " «Ils mangent les hommes, c'est dans la nature du tigre » répondirent-ils, alors l'empereur prit une flèche et un arc des mains de l'un de ses gardes et tua le fauve. Une fois le tigre mort, Kangxi réprimanda ses gardes : "Vous rencontrez un danger et vous ne réfléchissez pas, la plupart des tigres s'enfuient en descendant la montagne, celuici descendait pas à pas, il nous avait sûrement déjà rencontrés, comment pouvez-vous dire qu'il attaque l'homme? ». Les gardes, entendant cela, reconnurent qu'il avait raison, et pour exprimer leur regret, portèrent le tigre dans la salle principale du monastère et l'étendirent sur une table d'offrandes particulière (Wei Guozuo [1988] 1993, pp. 194-195).

En contexte bouddhique, même pour un saint empereur, chasser sur une montagne sacrée, a fortiori un tigre "inoffensif », est un péché, source de démérites. Dans une autre histoire chinoise provenant également du Wutaishan, Kangxi évita de répéter cette erreur et empêcha même que ses gardes ne tirent sur le renard-bodhisattva :

Lors de son troisième voyage en 1698, Kangxi arriva au Tailusi et vit un renard. Un de ses cavaliers s'apprêta à tirer une flèche mais Kangxi arrêta son bras, se rappelant l'histoire du tigre. Le renard observa les cavaliers et sembla vouloir qu'on le suive, il courut devant puis s'arrêta brusquement, vérifiant que l'empereur le suivait. Finalement Kangxi le suivit seul et lorsqu'il arriva à un petit temple en ruine au pied d'un grand arbre, le renard disparut. Kangxi comprit que le renard n'était autre que Mañjuśrī souhaitant attirer l'attention sur ce site, et fit restaurer le temple (il s'agit du Wenshusi 文殊寺) (Yang Zengwu 2005, pp. 182-183).

\section{Kangxi, l'arhat au tigre}

Est-ce en expiation de ses chasses que Kangxi est représenté par les Mongols accompagné d'un tigre, ou au contraire, parce qu'il a su dompter le tigre-démon ? Le fait est que des Mongols laïcs identifient parfois le dix-huitième arhat (représenté dans tout monastère mongol), accompagné d'un tigre dompté, à l'empereur Kangxi (fig. 6) ${ }^{38}$. Cet arhat est en fait dans le panthéon tibétain le dévot laïc Dharmatrāta (Dhar-ma ta-la) habillé à la mode d'Asie centrale avec bottes et pantalon ${ }^{39}$.

\section{Kangxi, le Haroun-al-Rashid mandchou}

\footnotetext{
${ }^{37}$ Lors du pèlerinage au Wutaishan qu'il fit avec sa grand-mère en 1683, Kangxi interdit expressément de prendre toute vie sur la montagne (Köhle 2008, p. 91).

${ }^{38}$ Identification que l'on retrouve dans des catalogues d'exposition (Narantuya 2005 : 6-7). Le « couple» formé par les deux arhat Dharmatrāta et Hva-shang n'est pas sans rappeler le «couple » formé par Dugar Jaisang, laïc pieux terrassant un tigre à mains nues, symbole des traditions hétérodoxes, et le Vieillard Blanc, peint à l'entrée des monastères mongols. Dans son récent article sur Dugar Jaisang (qui présente de nombreux points communs avec Dharmatrāta), C. Humphrey (2007) n'a toutefois pas évoqué d'association avec Kangxi.

${ }^{39}$ Son allure de pèlerin, avec son chasse-mouches et des sutras sur le dos, rappelle encore le personnage de Xuanzang 玄焋 (qui voyagea en Inde de 629 à 645), accompagné d'un tigre dans nombre de ses représentations.
} 
Les histoires mentionnées précédemment montrent que les Mongols attribuaient à Kangxi des pouvoirs divins. Pourtant, loin de se comporter comme un empereur lointain et intouchable, cloîtré dans sa Cité Interdite, il se montrait humain et proche du peuple. Kangxi était réputé pour son style direct et simple tant dans l'écriture que dans l'administration et les relations humaines. Il n'hésitait pas, en particulier lors de ses tournées d'inspection pour «observer le peuple» (guanmin 觀民) ${ }^{40}$, à quitter les gens de sa suite pour parler avec des locaux, pêcher avec des pêcheurs, et interroger les paysans sur les récoltes ${ }^{41}$. On lit dans un recueil d'anecdotes chinoises que l'empereur préconise « qu'il faut tout voir par soi-même » (Spence 1966, p. 131). Les missionnaires chrétiens ont loué sa grande diligence à écouter les plaintes, à redresser les torts et à punir ses fonctionnaires coupables $^{42}$. Lors de ses périples en Mongolie, il logeait chez l'habitant, partageait le koumiss dans les tentes mongoles, offrait à boire de ses propres mains et discutait avec les petites gens de leur vie et de leurs troupeaux. En ville, il distribuait de la nourriture aux gens qui s'assemblaient sur son passage. Il écoutait les plaintes, les remontrances et les pétitions (Spence 1974, pp. 17, 43) : il était si populaire que (d'après ses propres écrits) les gens le suppliaient de rester un jour de plus, ce qu'il acceptait à chaque fois (Spence 1966, p. 133). Il se montrait également très attentionné envers ses gens. En campagne, il pensait au bien-être de ses troupes dans le plus grand détail et protégeait les civils en temps de guerre (Spence 1974, pp. 16-17).

Cette image qu'il cherchait à donner suscita en Chine et en Mongolie de nombreux récits. Ceux-ci se confondirent avec des légendes qui circulaient en Asie centrale sur le calife Haroun-al-Rashid (768-809), popularisées par les Mille et une nuits. Comme le calife abbasside, Kangxi, las de la pompe de son palais, se déguise en homme ordinaire, en voyageur commerçant, pour "prendre le pouls » de son peuple. Il recherche les talents cachés pour les récompenser de ses grâces, et teste la loyauté de ses fonctionnaires.

\section{Kangxi et son âne}

La chronique mongole Bolur toli («Miroir de cristal») écrite par Jimbadorji au milieu du XIX ${ }^{\mathrm{e}}$ siècle relate l'entrée de l'empereur Kangxi à Pékin sur le dos d'un âne noir lors de sa première rencontre avec le ${ }^{\text {er }}$ lCang skya quturtu Ngag dbang blo bzang chos ldan $(1642-1715)^{43}$ :

Après la mort de l'empereur Shunzhi [1638-1661 ; r. 1644-1661], le 1Cang skya rencontra Kangxi dans le temple du Bouddha de Santal à Pékin. Kangxi lui demanda qui il était et d'où il venait. Comprenant qu'il avait affaire à un grand sage, il lui demanda s'il y avait des signes qu'il allait devenir empereur. Le lCang skya qutuүtu lui répondit : « Si tel mois, tel jour à telle heure, tu montes un âne noir et entres par telle porte, tu règneras en tant qu'empereur ». Kangxi promit au prélat de le vénérer de génération en génération si cela

\footnotetext{
${ }^{40}$ Sur les objectifs multiples des tournées d'inspection des empereurs Qing (en particulier de Qianlong dans le sud de la Chine), ainsi que la perception et les réactions du peuple et des lettrés : Chang 2007. Sur la tradition des empereurs chinois d' « observer le peuple »: Chang 2007, chap. 7.

${ }^{41}$ Par exemple Qingbai leichao [1917] 1984-1986, I, pp. 337-339.

${ }^{42}$ Par exemple Bouvet [1699] 1940, pp. 16-19.

${ }^{43}$ Kangxi nomma par la suite le prélat Précepteur d'État et lui confia d'importantes responsabilités politiques, diplomatiques et religieuses ; il devint le chef spirituel de la Mongolie-Intérieure.
} 
se réalisait. « À l'heure dite du jour dit, le prince (Kangxi) trouva un âne noir, le monta et entra par la porte arrière. À ce moment un grand bruit éclata dans le ciel et une pluie diluvienne tomba. Tous les gens à l'intérieur s'écrièrent: "Voici un dragon à cheval sur un âne !". Le bruit s'intensifia encore et encore et se propagea jusqu'à l'intérieur du palais. Tous crièrent: "Si un dragon vient, il vient certainement afin de s'assoir sur le trône impérial". Alors ils le revêtirent d'une robe et d'un chapeau appropriés, [...] puis ils l'assirent sur le trône impérial et à la fin de la cérémonie lui donnèrent le titre honorifique de Engke amurulang $\gg{ }^{44}$.

La tradition rapporte que derrière l'aile sud de la Porte de l'Harmonie (Xiehemen 協和 門), une porte de la Cité Interdite, une courte chaîne attachée à un pilier remuait toujours légèrement même sans un souffle de vent : on y attachait le petit âne noir de Kangxi quand il sortait incognito en excursion (Arlington et Lewisohn [1935] 1987, p. 34).

Les histoires de Kangxi parcourant l'empire incognito sur son âne se retrouvent chez les Ordos (Temür et al. 2009), chez les Tümed et chez les Qorcin. C'est certainement parce que l'empereur aimait chasser dans les Ordos que les Mongols de cette région colportèrent de nombreuses histoires à son sujet ${ }^{45}$. Le vieux conteur ordos Co $\gamma_{r u b}{ }^{46}$ présente Kangxi comme l'empereur qui a pacifié tous les royaumes, et donne une explication de l'origine de son âne merveilleux :

Kangxi resta une année chez un couple de vieux mendiants ; il traitait le vieux mendiant comme son frère juré et l'invita à Pékin pour son anniversaire. En route pour Pékin, le vieux, qui n'avait pour tout cadeau à offrir qu'un sac de jujubes, trouva dans une famille « un animal de couleur gris-âne au corps ressemblant à une souris, avec de longues oreilles ». Ce dernier était apparu miraculeusement lorsque, afin d'en tirer de la soupe, l'on cassa et fit bouillir une planche en bois qui avait servi à un boucher d'ânes. L'animal suivit le vieux mendiant qui le mit dans sa poche, mais quand celui-ci sortit l'animal de sa poche en disant «si seulement cet animal était un âne !", l'étrange bête devint un gros âne gris, grâce auquel il put arriver à temps à la fête.

Kangxi accueillit le vieux en vêtements civils et lui fit traverser les immenses cours du palais, mais c'est seulement lorsqu'il mit ses vêtements d'empereur que le vieux comprit que son frère juré était son souverain. Il offrit son sac de jujubes à Kangxi qui se réjouit, parlant des jujubes comme le meilleur des fruits : c'est depuis ce jour que l'on a coutume d'offrir des jujubes rouges à chaque rituel. Le lendemain, les tributaires offrirent à l'empereur des brassées d'or et d'argent, des joyaux et des perles, des soies et des brocards, mais le vieux n'avait quant à lui plus rien à offrir. Il pensa alors à son âne, et le fit parvenir à l'empereur qui se réjouit car «il n'appartient pas au bétail des cinq museaux $^{47}$, c'est une monture qui ne se compare pas à la soie et aux joyaux ». L'âne s'avéra être une monture extraordinaire, capable de parcourir 1500 yazar (750 km environ) par jour. Kangxi fit également venir la vieille mendiante à Pékin, et le couple de mendiants vécut heureux jusqu'à la fin de sa vie tandis que l'âne devint le compagnon des

${ }^{44}$ Bolur toli, II, fol. $32 \mathrm{v}-34 \mathrm{v}$, texte et traduction en allemand par Sagaster 1967, pp. 349-351. Sagaster rapproche cette histoire de celle de la prophétie de l'entrée de Jésus à Jérusalem et soupçonne les Jésuites de Pékin d'en être à la source.

${ }^{45}$ Voir les lettres écrites par Kangxi lui-même (Cimeddorji 1991, pp. 209-210) ainsi que le témoignage du père Gerbillon (Du Halde 1735, IV, p. 346).

${ }^{46}$ Je n'ai découvert qu'en terminant d'écrire cet article que Walther Heissig (1999) avait déjà résumé les histoires de Coyrub à partir de la version publiée dans le Ordos-un soyul-un öbür-ün cuburil 4, p. 108. Je n'ai pas trouvé ce livre ; la version donnée par Temür et al. (2009) présente quelques différences. Je me contente ici d'en rappeler très brièvement le contenu et de détailler ce qui diffère de la version traduite par Heissig.

${ }^{47}$ Chevaux, moutons, chèvres, vaches et yaks, et chameaux. 
expéditions incognito de Kangxi à travers le pays (Temür et al. 2009, pp. 177-180; voir aussi Heissig 1999, p. 78).

Les Mongols ont pu inventer cette histoire pour justifier le fait que Kangxi monte un âne - en Chine, la monture du commerçant, du pauvre, de l'homme du commun, et pour les Mongols, une monture inférieure, indigne des cavaliers de la steppe. Or l'animal s'avère être un âne exceptionnel, digne d'un empereur, capable de parcourir 1500 razar par jour (les fabuleux chevaux du Ferghana tant convoités par les Chinois parcouraient, dit-on, 1000 li (environ $500 \mathrm{~km}$ ) par jour.

\section{Kangxi déguisé teste la loyauté de ses fonctionnaires}

D'autres histoires relatent que Kangxi se mêlait à la foule et enquêtait incognito sur les méconduites de l'administration impériale, les joies et les souffrances du peuple, afin de lutter contre l'injustice, la tyrannie, l'oppression et l'exploitation. Chez les Qorcin, on emploie le terme de sifangla- (ch. sifang 私访, «mener incognito une enquête personnelle $»)^{48}$ (communication de B. Uranchimeg, 7 novembre 2011). Coyrub raconte encore :

Kangxi parcourant le pays arriva à proximité de la Ville des Chameaux (nom populaire mongol de Yulin 榆林, au Shaanxi). [...] Mais alors qu'une tempête de neige faisait rage, il trouva closes les portes de Yulin. Il tenta alors de persuader le garde de les ouvrir en lui offrant 50 taels d'argent; ce dernier refusa le pot-de-vin et répondit en se moquant: «Cette porte, est-ce une porte pour toi et moi [seulement] ! J'ai un ordre de l'empereur, je l'ouvre à l'heure, et quand l'heure (de la fermer) est arrivée je la ferme, c'est une porte qui est régie par un règlement ». L'empereur loua son comportement et s'en alla (Temür et al., 2009, pp. 181 ; voir aussi Heissig 1999, p. 79).

De nombreuses histoires semblables circulent en Chine, qui toutes confirment le strict respect de la loi par les soldats de l'empire (la lutte contre la corruption étant un souci principal du règne de Kangxi). L'empereur, dit-on, vérifiait l'étanchéité de ses frontières en voyageant à la Passe Shanhai 山海關 (Hebei) déguisé en marchand de chapeaux pékinois. Les gardes, suspicieux, le giflèrent et le renvoyèrent. Lorsque Kangxi voulut récompenser leur diligence, ils se pendirent en apprenant qu'ils avaient battu le fils du Ciel (Lovell 2006, p. 259).

\section{Kangxi goûte les spécialités régionales}

Malgré le manque de valeur apparente d'un sac de jujubes, l'empereur en fait, comme on l'a vu ci-dessus, une offrande de choix dans les rituels. D'après une histoire qorcin :

Kangxi (Amurulang Ejen) voyageait incognito sur son âne, rentrant à Pékin. Un jour où il était particulièrement affamé, il pénétra dans une maison où se trouvait une vieille femme et lui demanda quelque chose à manger. La vieille lui prépara des beelmien ${ }^{49}$ beeb (wowotou 窝窝头, pain de maïs à la vapeur), qu'il trouva délicieux. Kangxi lui demanda le nom de ce plat; elle lui répondit qu'il s'appelait lierwaiba 里二外八, ce qui signifie

\footnotetext{
${ }^{48}$ Le dialecte qorcin comprend de nombreux termes venant du chinois.

${ }^{49} \mathrm{Du}$ russe pel'men, pain/ravioli à la vapeur?
} 
« [que ]'on place] deux [doigts] à l'intérieur et huit à l'extérieur » lors de la fabrication de ce pain. De retour dans son palais, l'empereur ordonna à ses cuisiniers de confectionner des lierwaiba, mais aucun de ces pains n'avait exactement le même goût et n'était aussi bon que les lierwaiba pétris par la vieille femme. Aussi il retourna voir la vieille dame pour qu'elle lui en refasse. Cependant, il ne les trouva pas aussi bons que la première fois. La vieille dame expliqua que c'était parce qu'il était affamé qu'il les avait trouvés si bons la première fois (histoire racontée par Uranchimeg, 7 novembre 2011).

Ou encore, dans une histoire ordos, arrivant à proximité de Yulin,

il y loua la qualité des pastèques, et depuis ce jour les gens de Yulin se vantent de cultiver les meilleures pastèques de la région (Temür et al. 2009, pp. 180-181; voir aussi Heissig 1999, p. 79).

De même, dans un village sur la route de Yinchuan 銀川 (Ningxia), il goûta d'excellents pains à la vapeur qui, depuis, sont devenus réputés (ibid.). L'empereur gastronome, bien que voyageant incognito, aurait confirmé ou révélé la qualité des produits régionaux.

On retrouve une histoire similaire au Wutaishan, où un plat, improvisé en raison de l'absence du cuisinier, fut servi à l'empereur Qianlong qui, le trouvant excellent, en demanda le nom : ce serait l'origine des «oreilles de chat» (貓耳朵), nouilles en forme d'oreilles de chat, semblables aux orecchiette italiennes (Yang Junlei 2008 p. 175).

\section{L'empereur en danger : légendes tümed}

Parcourir le pays incognito et sans escorte n'est naturellement pas sans danger. Dans les histoires suivantes des Tümed de Hohhot, l'empereur se retrouve dans une situation délicate qui le met en position d'infériorité : il ne cherche pas à rendre justice mais à

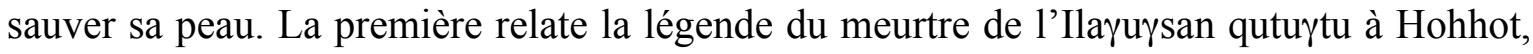
rapportée dans des sources chinoises et mongoles comme dans des récits de voyageurs occidentaux.

Kangxi profita de son expédition militaire contre les Züüngar pour venir saluer le dignitaire religieux dans son monastère. Or ce dernier pratiquait un jeûne lors duquel il ne

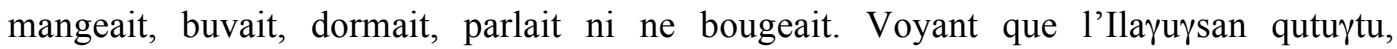
immobile, ne rendait pas les honneurs à Kangxi, un officier de la suite impériale, le général Bai, s'emporta et tua le lama. Les moines en colère massacrèrent les officiers de l'empereur mais Kangxi parvint à s'enfuir et se cacha au Baya zuu. Il y laissa son armure et se déguisa en simple soldat, en lama ou en marchand (selon les versions), pour s'enfuir incognito vers Kalgan (ch. Zhangjiakou). Un bosquet de saules roses apparut miraculeusement à Yusuling à l'est de Hohhot où l'empereur poursuivi put se réfugier. Selon Pozdneev, le général Bai fut ensuite tué par les moines ou se suicida, et les Chinois érigèrent un temple en son honneur (Guisui xian zhi [1934] 1968, p. 304 ; Pozdneev [1896-1898] 1977, p. 44).

Kangxi n'est pas directement responsable de la mort du grand lama ; c'est un incident diplomatique regrettable, et c'est à son habitude de se fondre dans le peuple qu'il doit la vie. L'histoire est aussi rapportée dans un recueil d'anecdotes dans lesquelles c'est le duc Fei Nangzhuang 費囊莊 qui aurait exécuté le lama ${ }^{50}$. Elle est encore racontée par le père

\footnotetext{
${ }^{50}$ Xiaoting xulu [XVIII ${ }^{\mathrm{e}}$ siècle] 1996, chap. 2, p. 618 : « Fei Nangzhuang zhi sha huofo » 費囊莊之殺活佛.
} 
Huc - qui appelle le lama « Guison-Tamba » (Jebcündamba) et situe la scène aux " Cinq Tours » (monastère Tabun suburyan-u süme ou Wutasi 五塔寺, Hohhot) ${ }^{51}$. Elle est enfin reprise dans plusieurs autres sources occidentales ${ }^{52}$. D'après une version, pour se faire pardonner, Kangxi aurait fait construire le Temple Jaune de Pékin (en réalité construit par son père) (Bredon 1922, p. 224). Le récit ci-dessus fournit aussi une explication à la présence de (ou des) l'armure(s) de Kangxi au Baya zuu.

Le Père Gerbillon, témoin direct qui accompagnait la délégation de Kangxi, rapporte la visite de l'empereur à un lama réincarné de Hohhot en 1688 (Du Halde 1735, IV, p. 103), mais n'évoque aucun incident de ce genre ; il remarque au contraire que les grands lamas, puisqu'ils font obédience à Bouddha, sont dispensés des prosternations à l'occasion de la

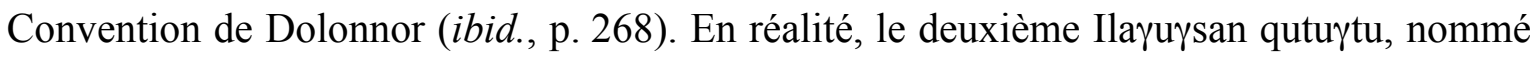
en 1685 administrateur (jasa $\gamma$ da blama) du Bureau des Sceaux (administration des affaires religieuses) de Hohhot et envoyé comme ambassadeur auprès des Züüngar en 1690-1692, avait trahi l'empire et rallié leur chef, Galdan. Il fut capturé, et exécuté dans le Temple Jaune de Pékin en 1697 devant les princes mandchous et mongols de la capitale et les fonctionnaires civils et militaires ${ }^{53}$. La scène de meurtre du pontife a peut-être été inventée

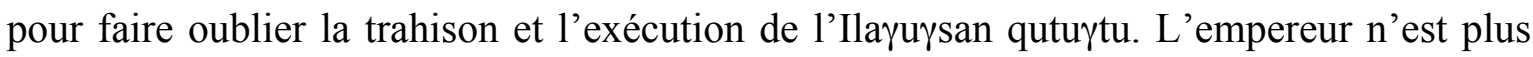
celui qui donne l'ordre d'exécuter un traitre mais au contraire celui qui doit subir les conséquences du geste impulsif d'un de ses officiers.

La deuxième histoire est illustrée par une peinture narrative de 3,50 mètres de long, nommée «Le Pavillon de la lumière lunaire », Yueminglou 月明樓, qui date des années 1889-1893 (fig. 7). Elle était jadis exposée dans une salle du Jeke zuu («Grand monastère ») de Hohhot. L'empereur Kangxi y figure, entouré de 110 personnages, parmi lesquels on distingue des Mongols (identifiés par leurs bottes et leurs vêtements), des Chinois et des divinités bouddhiques. La peinture illustre l'histoire suivante :

Kangxi, lors d'une de ses campagnes contre les Oirad, s'arrêta dans une ville et, déguisé en marchand, se rendit dans une auberge nommée Yueminglou pour dîner. Le montant de l'addition excédant largement l'argent qu'il avait sur lui, il accusa l'aubergiste de chercher à l'extorquer. Une bataille s'ensuivit, dont le vacarme réveilla un certain Xiao Liusan 小 劉三, employé de l'aubergiste, qui vola au secours de l'empereur déguisé, et demanda à l'aubergiste son année de salaire pour payer l'addition. Xiao et Kangxi firent alors un serment de frères jurés, et, de retour à la capitale, l'empereur convoqua Xiao pour le nommer fonctionnaire du quatrième degré (Sai Wendusu 1961, p. 63).

La peinture représente Xiao Liusan levant la main gauche pour recevoir son dû, entouré de personnages en train de regarder, de se battre ou de manger. On retrouve ici le thème de l'empereur récompensant la générosité et la compassion d'un homme du commun. Or le

\footnotetext{
${ }^{51}$ Huc [1924] 1987, I, p. 215.

${ }^{52}$ Baddeley 1919, II, p. 166 ; Markham (dir.) 1876, pp. xlviii-xlix (d'après Huc) ; Waddel [1895] 1939, pp. 240-241 (citant Markham). Le Jebcündamba n'a jamais résidé à Hohhot, et le monastère des «Cinq

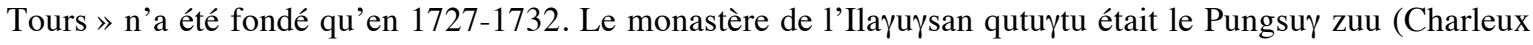
2006, [9]).

${ }^{53}$ Références dans Spence 1974, p. 31.
} 
Jeke zuu était le lieu d'un culte impérial : ce monastère conservait un trône réservé à l'empereur mandchou et une tablette en lettres d'or proclamant « longue vie à l'empereur ». Le premier jour du premier mois lunaire, et ce jusqu'à la fin des Qing, les fonctionnaires mongols, mandchous et chinois de Hohhot se prosternaient devant la tablette impériale, et les moines exécutaient un rituel destiné à assurer longue vie à l'empereur. Il est intéressant de trouver dans le même monastère un culte à l'empereur divinisé et une peinture illustrant un épisode de Kangxi dans une situation difficile.

\section{L'empereur ignorant des coutumes}

Un empereur ne sait pas toujours comment on doit se comporter parmi le peuple. Kangxi commet des erreurs par ignorance ou négligence, mais sait les reconnaître et se repent, demande pardon et fait preuve d'humilité, sans chercher à se venger ou à profiter de sa position.

Arrivé dans une petite ville des environs de Xi'an (Shaanxi), Kangxi prit un repas mais au moment de payer, il fit tomber une petite pièce trouée sans valeur et ne prit pas la peine de la ramasser. Voyant cela, le serveur l'admonesta : « Les pièces de monnaie appartiennent à l'empereur! Pourquoi l'écrases-tu avec ton pied?». Kangxi loua alors ce peuple vertueux qui respecte la propriété de l'empereur.

Continuant son chemin, il atteignit le grand monastère de Kumbum (en Amdo au Tibet oriental) et pénétra dans la cour sans descendre de son âne. Le cuisinier (ou selon une autre version le porteur de bois du temple) le frappa cinq fois avec un balai ou un fagot. Kangxi descendit de son âne, reconnut son erreur et lui offrit respectueusement cinq fouets noirs. C'est ainsi que l'on explique la dureté des règles de Kumbum (Temür et al. 2009, pp. 181-182 ; voir aussi Heissig 1999, p. 79-80).

Bien que faisant preuve d'une extrême humilité, jusqu'à rester un an avec un couple de mendiants sans être découvert, Kangxi commet toutefois des impairs compte tenu de son rang et fait preuve de mépris pour les coutumes locales. Le «petit peuple » lui donne des leçons d'humilité, de respect des coutumes, et n'hésite pas à l'insulter, à se moquer de lui ou même à le battre. Ces récits sont une leçon de morale sur l'importance de respecter les traditions locales, les règlements impériaux (sur l'heure de fermeture d'une ville), et l'argent, propriété de l'empereur. Ils louent l'honnêteté, le sens du devoir et la loyauté du petit peuple (souvent chinois dans ces histoires mongoles) qui observe scrupuleusement règlements et coutumes, et qui possède des biens plus précieux que l'or, l'argent et les brocarts : des jujubes, un âne magique, des pastèques excellentes. Ces légendes fournissent des explications sur l'origine de traditions locales (les offrandes de jujubes, le dur règlement de Kumbum). Enfin, elles permettent de se moquer des personnes haut placées qui pensent être au-dessus des règles. L'empereur apparaît dans ces histoires comme naïf et profondément humain. On est loin de l'image de l'empereur chasseur et conquérant, mais on retrouve le sens de la justice et la générosité des récompenses.

Ce même type de récit circulait non seulement parmi les Mongols, mais aussi parmi les Chinois à l'époque moderne. L'anecdote suivante, par exemple, provient du Wutaishan. 
Kangxi déguisé en marchand aisé, accompagné de deux eunuques, se rendit dans un magasin du village de Taihuai 臺懷 et admira une exceptionnelle calligraphie d'enseigne de boutique. Il voulut récompenser le calligraphe en lui donnant un poste. Mais en regardant en détail, l'empereur s'aperçut que le calligraphe avait signé «roi des caractères 》(Ziwang 字王). Scandalisé par son arrogance, Kangxi voulut donner une leçon à l'impertinent, avant d'apprendre qu'il s'agissait simplement de son nom, Wang Zi, ainsi appelé parce qu'il était le seul à savoir lire dans sa famille. L'empereur octroya au calligraphe une fonction à l'académie Hanlin 翰林院 de Pékin (Yang Zengwu 2005, pp. 112-113).

\section{Le redresseur de torts qui se trompe de cible}

Dans deux récits chinois rappelant ceux du souverain déguisé parcourant son pays, Kangxi se pose en défenseur de la moralité et de l'orthodoxie bouddhique ${ }^{54}$ mais se trompe de cible. Ces histoires proviennent du folklore du Wutaishan.

L'empereur aperçut un moine et une femme se baignant nus dans un bassin ; il banda son arc et décocha une flèche pour abattre le moine au comportement scandaleux, mais celuici parvint à s'enfuir. Kangxi suivit les traces de sang qui aboutissaient au Pusading 菩薩 頂, principal monastère tibéto-mongol de la montagne sacrée, entra dans une salle du monastère et vit une statue de Mañjuśrī transpercée d'une flèche dans l'épaule droite : c'était donc sur le bodhisattva en personne [dont lui-même est considéré comme une émanation], qu'il avait tiréén

Les pèlerins viennent encore voir cette célèbre statue du Pusading, appelée Mañjuśrī à la Flèche (Daijian Wenshu 帶箭文殊). L'anecdote est aussi représentée sur la carte mongole (où Mañjuśrī se baigne avec deux femmes). Si le début de l'histoire est calqué sur une légende plus ancienne ${ }^{56}$, la fin a probablement été inventée par la suite. Dans une autre histoire,

Kangxi réduisit en cendres le Shuxiangsi 殊像寺 où une princesse mandchoue, fille de Shunzhi faite nonne, avait été vue discutant les sutras avec un jeune moine. Mais tous deux furent retrouvés sains et saufs après l'incendie, preuve que leur relation était purement intellectuelle. Pour racheter sa faute, Kangxi fit rebâtir le monastère (Wei Guozuo [1988] 1993 : 105-106).

Les récits chinois et mongols suivent généralement la même trame : l'empereur arrive dans un lieu inconnu, admoneste quelqu'un ou commet une erreur par inadvertance, puis reconnaît son tort et offre une réparation (poste à la cour, construction d'un monastère).

\section{Kangxi et le Sceau de Jade}

\footnotetext{
${ }^{54}$ Ce qui le rapproche de Dugar Jaisang, voir note 38.

${ }^{55}$ Voir une autre version dans Chou 2007, p. 124. D'autres légendes du Wutaishan relatent la rencontre de l'empereur avec le bodhisattva ayant pris une forme humaine ou animale : par exemple l'histoire du vieux moine à la lanterne du Jixiangsi 吉祥寺, elle-même reprise d'une histoire plus ancienne où le protagoniste principal n'est pas Kangxi mais le moine Biyun 碧雲 (Wei Guozuo [1988] 1993,p. 142).

${ }^{56}$ Un certain gouverneur Li Jing 李靖 de l'époque Song, aurait tiré sur un moine se baignant avec une femme, qui se révélèrent n'être autres que Mañjuśrīet Samantabhadra (Chou 2007, p. 124).
} 
D'après une histoire qorcin :

Kangxi voyageait incognito sur son âne, à la recherche de son Sceau de Jade. Il entendit dire que son père (l'empereur Shunzhi) était au Wutaishan (mong. Utai). Il se rendit au Wutaishan, et après avoir interrogé de nombreuses personnes, il arriva à une grotte où il pensait que son père méditait. Il s'agenouilla et se prosterna à l'entrée de la grotte, appelant «père » la personne qui se trouvait à l'intérieur. L'homme répondit " Je ne suis pas ton père. Toutefois, maintenant que tu m'as appelé "père" et que tu t'es prosterné devant moi, je vais te donner le Sceau de Jade de l'État». En fait, il apparut que cet homme était Li Zicheng ${ }^{57}$ qui s'était rebellé contre l'État Ming et s'était emparé du Sceau de Jade. Lorsqu'il avait été vaincu, il avait emporté le sceau avec lui au Wutaishan, où il s'était fait moine (histoire racontée par Uranchimeg, 7 novembre 2011).

Cette histoire originale amalgame différentes légendes. Au Wutaishan, de nombreux récits portent sur Kangxi à la recherche de son père, l'empereur Shunzhi, qui aurait fait proclamer l'annonce de sa mort afin de se retirer dans un monastère de la montagne ${ }^{58}$. Par exemple :

Kangxi aperçut un vieux moine qui balayait la cour du monastère Zhenhaisi 鎮海寺; celui-ci disparut en écrivant son nom : Bafu 八X sur le mur. Kangxi comprit trop tard que les deux caractères combinés formaient le mot "père» ( $f u$ 父) (Yang Zengwu 2005, p. 181).

Dans une autre histoire chinoise :

Kangxi vit un poème écrit sur un mur du Shancaidong 善財洞 (ou du Tailusi) et le recopia. De retour à Pékin, sa mère reconnut la calligraphie de Shunzhi. Kangxi retourna chercher le moine auteur du poème au Wutaishan mais il venait de décéder (Yang Zengwu 2005, pp. 181,187$)^{59}$.

Le thème de Kangxi cherchant son père est toujours très présent au Wutaishan (bien qu'il ne figure pas sur la carte destinée aux pèlerins mongols). En fait, si les Chinois aimaient particulièrement ces histoires qui étaient prétexte à des jeux de mots ou expliquaient l'origine de poèmes écrits sur des murs, l'histoire qorcin en reprend la trame générale, celle de la recherche du père disparu, mais la véritable quête est celle du Sceau de Jade. Il s'agit du célèbre Sceau de Jade qui Transmet l'Empire (chuanguo xi 傳國爾), objet mythique et magique, symbole du Mandat Céleste et donc outil de légitimation. L'histoire de ce sceau, qui aurait été forgé pour le premier empereur de Chine, Qin Shihuang, réapparaît à chaque fois qu'une dynastie est jugée usurpatrice (Aubin 2010). D'après les chroniques mongoles du XVII ${ }^{\mathrm{e}}$ siècle, le sceau réapparut entre les mains de Gengis Khan, puis fut transmis à ses successeurs, de Khubilai Khan à Ligden Khan. Si l'on en croit l'historiographie mandchoue, le souverain Hung taiji se serait approprié le sceau de jade impérial de la dynastie Yuan à l'occasion de sa victoire sur les Caqar en $1634^{60}$.

Dans notre histoire, Kangxi n'est pas encore en possession du sceau mais le recherche et le considère comme sien : puisqu'il a déjà le Mandat du Ciel, il est un empereur légitime

\footnotetext{
${ }^{57}$ Leader de la rébellion paysanne contre les Ming, Li Zicheng 李自成 (1606-1645) proclama une dynastie en avril 1644 mais fut défait le mois suivant par les Mandchous. Les circonstances de sa mort sont inconnues, mais la tradition populaire s'empara de sa légende : on dit qu'il survécut à ses défaites et se retira dans un monastère.

${ }^{58}$ Cette légende a connu de nombreux développements. L'étude des sources mandchoues a toutefois montré qu'il serait mort de la variole en 1661.

${ }^{59}$ Voir également Fang Qingqi et al. 1985.

${ }^{60}$ Weiers (2000) a montré comment Hung taiji inventa de toutes pièces l'histoire de sa récupération du sceau de jade afin d'appuyer ses visées politiques en 1635.
} 
et le sceau doit naturellement lui revenir ${ }^{61}$. Sans savoir qu'il s'adresse à l'empereur, Li Zicheng le lui remet parce qu'il se comporte en fils pieux. Kangxi devient donc héritier de Chinggis Khan et de Khubilai Khan via Li Zicheng, perçu par les Qorcin comme un empereur légitime (ce qu'il n'est pas dans l'historiographie chinoise). Les histoires chinoises de piété filiale se sont ainsi transformées en histoires mongoles de quête de légitimité politique.

\section{Kangxi se méfie de l'astrologie}

Selon une histoire qorcin :

Lorsque Kangxi voyageait incognito sur son âne en direction de Pékin, il arriva dans une famille où se tenait une noce. Sachant que c'était un jour néfaste pour le mariage, il alla trouver le devin pour lui demander pourquoi il avait choisi cette date. Ce dernier répondit qu'il le savait, mais que le caractère néfaste serait changé en bénéfique parce que le fils du Ciel allait visiter cette famille. Kangxi, abasourdi et craignant d'être reconnu, quitta immédiatement la fête sans avaler un morceau. C'est de là que viendrait le terme

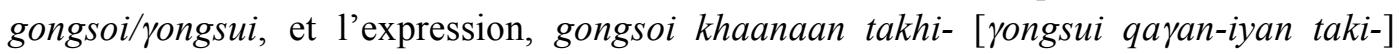
("vénérer l'empereur pour rien »). Le verbe yongsui- décrit la déception d'une personne qui ne parvient pas à trouver ce qu'elle cherche (histoire racontée par Uranchimeg, 7 novembre 2011).

Cette histoire racontée chez les Qorcin se retrouve chez les Ordos :

Arrivant au sein d'une famille en train de célébrer un mariage, Kangxi qui voyageait incognito s'étonna qu'un jour réputé néfaste fut choisi pour la fête. Il demanda au maitre de maison pourquoi ce jour avait été choisi, et apprit que l'on avait utilisé le livre de l'astrologie jaune (sira ziruqai) ${ }^{62}$, selon lequel c'était un jour faste puisque c'était le jour de la venue de l'empereur. Rentré à Pékin, Kangxi décréta l'interdiction stricte de l'utilisation de l'astrologie jaune (Temür et al. 2009, pp. 182 ; voir aussi Heissig 1999, p. 81).

Différentes traditions mongoles concernent l'interdiction de la divination au moyen de tableaux astrologiques ou autres méthodes (l'une d'elles étant même attribuée à Chinggis Khan) sous prétexte qu'elle peut mener à de mauvaises décisions et entraîner désillusion et colère $^{63}$. Ces traditions sont ici transposées sur la personne de Kangxi, mais on comprend

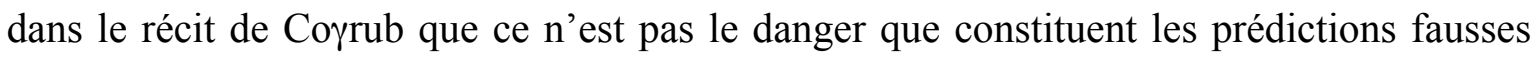
qui est visé mais au contraire leur exactitude, d'autant plus dérangeante que l'empereur était déguisé.

\section{Conclusion}

Les actes de l'empereur Kangxi parcourant son empire ont été couchés par écrit et déformés, tandis que des histoires ont été inventées ou ont fusionné avec des légendes plus anciennes, puis ont circulé en Chine et en Mongolie. Dans la majorité des cas, ces récits se

\footnotetext{
${ }^{61}$ D'après Bawden, « Mongol popular tradition denies that the seal ever came into the possession of the Manchus at all » ([1968] 1989, p. 47).

${ }^{62}$ Voir Heissig 1999, pp. 81-81.

${ }^{63}$ Sur ces différentes traditions : Heissig 1999, pp. 80-81 et notes.
} 
fondent sur une base historique et s'élaborent autour de valeurs et de motifs narratifs empruntés à différentes traditions orales. Kangxi apparaît dans certains récits comme un empereur juste et magnanime qui dispense ses grâces, un souverain chasseur et valeureux ; dans d'autres, comme un empereur soucieux du bien-être de son peuple et des malversations de son administration, s'élevant contre les injustices et louant la loyauté de ses sujets, récompensant les mérites et l'honnêteté des petites gens, mais se retrouvant parfois dans des situations délicates à cause de ses maladresses. Juge masqué, il récompense de façon démesurée les hommes de valeur, quel que soit leur statut, et traque ceux qui ne respectent pas la loi. Ce sont ces mêmes valeurs que les Mongols attribuent à Chinggis Khan qui punit impitoyablement toute trahison, mais tolère que l'on s'oppose à lui par loyauté envers un autre. Si Kangxi est perçu comme un patron du bouddhisme dans les danses masquées, cet aspect de l'empereur n'est pas mis en valeur dans les histoires.

On constate l'absence dans ces récits du Kangxi conquérant; la plupart des histoires se déroulent dans un empire en paix dont les activités les plus trépidantes sont la chasse et la bagarre dans les auberges. Serait-ce parce que l'empereur s'ennuie qu'il parcourt son pays incognito ? Pour les Mongols, le règne de Kangxi est vu a posteriori comme une période de paix - c'est d'ailleurs le sens même du terme Amurulang, nom mongol de Kangxi. Cela me semble être une indication de la date tardive de ces légendes, postérieures aux conquêtes de Kangxi, et peut-être à celles de Qianlong.

On ne peut savoir si les récits transmis oralement ou au travers de monographies locales $\mathrm{du} \mathrm{XX}^{\mathrm{e}}$ siècle ont été élaborés du vivant de Kangxi ou sont plus tardifs ; mais un certain nombre sont attestés au XIX ${ }^{\mathrm{e}}$ siècle, à la fin de l'empire. Certains, comme l'histoire qalqa rapportée par Cerensodnom, trahissent une influence bouddhique. Les histoires de l'empereur voyageant incognito semblent provenir d'un fonds chinois - elles se déroulent d'ailleurs souvent en Chine -, mais les Mongols les ont reprises et « télescopées » avec des histoires locales et des légendes originaires d'Asie centrale. Bien que ces histoires soient pleines d'un humour légèrement subversif se moquant des personnes de haut rang, l'image de Kangxi n'est jamais dégradée : les Mongols ne tournent jamais en dérision l'empereur mandchou et ne critiquent pas l'ordre établi. Au contraire, la grande longévité de ces récits qui se sont transmis jusqu'à aujourd'hui montre l'attachement que les Mongols ont conservé pour Kangxi. Il est dans tous les cas pleinement intégré au folklore des Mongols de Chine et de Mongolie comme une figure de conciliation des trois cultures, chinoise, mandchoue et mongole, et aucun autre empereur mandchou ou chinois ne jouit d'une semblable estime à leurs yeux.

En Chine Han, les anecdotes de l'empereur déguisé sont beaucoup plus fréquemment attribuées à Qianlong ${ }^{64}$, à l'exception des récits du Wutaishan. Les Mongols ont pu reprendre ou s'inspirer d'anecdotes chinoises en changeant le personnage principal. Certaines sont rassemblées dans un roman de la fin des Qing intitulé Qianlong xia Jiangnan 乾隆下江南 (ou Qianlong you Jiangnan 乾隆遊江南, “Qianlong descend/voyage au Jiangnan », s.d., probablement $\mathrm{XIX}^{\mathrm{e}}$ siècle) ${ }^{65}$, aujourd'hui popularisé

\footnotetext{
${ }^{64}$ Toutefois, un roman publié en 1916 et prétendument daté de 1701 relate des anecdotes sur les tournées de Kangxi dans le sud : Kangxi nanxun miji 康熙南巡秘記.

${ }^{65}$ Je remercie Vincent Durand-Dastès pour m'avoir indiqué l'existence de ce roman.
} 
par une série télévisée éponyme. Qianlong y joue les justiciers et s'entoure de redresseurs de torts. Et pourtant, les populations qui ont accueilli les tournées de Qianlong dans le Jiangnan, loin de la jubilation hystérique décrite dans les sources officielles, ont souffert d'abus de pouvoir et d'anxiété face à la montée des prix. En 1752 l'empereur a confisqué et interdit un ouvrage intitulé Nanxun lu 南巡錄 («Recueil sur les tours d'inspection dans le sud») qui discréditait ses voyages et le comportement de ses gardes (Chang 2005, chap. 3).

Comment se sont propagés ces récits ? On sait que les Mongols vivant à Pékin, et les Mongols qorcin, sinophones et souvent sinophiles, ont largement contribué à la circulation de la littérature chinoise écrite parmi les Mongols, en particulier de romans comme le «Pèlerinage vers l'Occident» (Aubin 1987; Atwood 1992-1993). Mais la propagation orale des légendes chinoises et leur développement proprement mongol a pu être le fait d'acteurs bien plus variés : les Mongols sinisés de Hohhot, les Qorcin et les Mongols résidant à Pékin ; les commerçants mongols parcourant l'empire, les pèlerins et marchands se rendant au Wutaishan, les Mongols au contact de commerçants chinois résidant ou voyageant en Mongolie, les princes mariés à des princesses mandchoues... Le Wutaishan, important point de contact sino-mongol, a certainement joué un rôle dans la transmission de ces récits. La carte du Wutaishan de 1846, dont plusieurs exemplaires ont été trouvés en Mongolie, atteste de la popularité de ces légendes auprès des Mongols. Enfin les Musulmans chinois qui commerçaient en Mongolie connaissaient aussi des légendes semblables et ont pu servir d'intermédiaire. Selon une légende populaire,

les conseillers de Kangxi voulant interdire la pratique de l'islam, dirent à l'empereur que les Musulmans se rassemblaient dans la principale mosquée de Pékin, rue du Bœuf, pour fomenter une rébellion. Kangxi se déguisa en musulman, pénétra dans la mosquée, mais ne voyant rien d'alarmant, émit par la suite un décret pour protéger la communauté musulmane (Li et Luckert 1994, pp. 222-228).

On peut aussi se demander dans quelle mesure certaines légendes issues ou non de faits réels ont été propagées volontairement par l'empereur et ses lettrés ainsi que ses successeurs à des fins de propagande impériale, dans le but de démontrer que les souverains mandchous étaient bien les héritiers de Chinggis Khan et de Khubilai Khan. L'historiographie Qing et en particulier le Shengwu ji de Wei Yuan glorifient les victoires militaires et la sagesse de Kangxi (Perdue 2005, p. 191). L'histoire du tigre tué au Wutaishan se retrouve ainsi dans plusieurs écrits de lettrés ayant accompagné l'empereur,

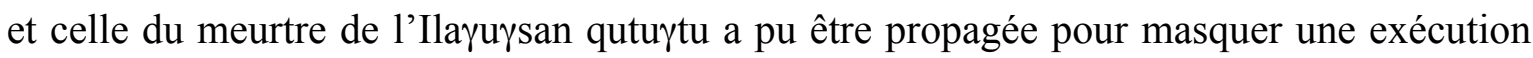
politique en accident regrettable afin d'apaiser le mécontentement des Mongols. Mais même si les Mandchous ont pu propager des histoires magnifiant leur image, célébrant leurs exploits et légitimant leur règne, rien ne permet de penser que les Mongols y ont adhéré. Il semble plutôt qu'ils aient fait des choix parmi ces légendes, retenant l'arrièreplan d'une histoire et greffant d'autres personnages ou d'autres quêtes, comme dans le récit de la transmission du Sceau de Jade.

Les récits sur Kangxi sont aujourd'hui très populaires en Chine, tant en littérature qu'en séries télévisées, cinéma et mangas. On mentionnera par exemple la série télévisée 
《Kangxi weifu sifangji » 康熙微服私訪記 (《Les voyages incognito de Kangxi ») (fig. 8), ou encore, le film de kong-fu intitulé «Kangxi danao Wutaishan»康熙大閙五臺山 (« Kangxi sème le désordre au Wutaishan », traduit en anglais sous le titre « The prisoner of Five Boulders »), dirigé par Yu Deshui 于德水 (Pearl River Film studio, 1989), dans lequel Kangxi part à la recherche de son père ${ }^{66}$. Ces films contribuent aujourd'hui à diffuser et augmenter la légende de Kangxi parmi les Mongols de Chine.

\section{Bibliographie}

Allsen, Thomas 2006 The Royal Hunt in Eurasian History (Philadelphia, University of Pennsylvania Press).

Arlington, L.C., et William Lewisohn [1935] 1987 In Search of Old Peking (Hong Kong/Oxford/New York, Oxford University Press) [Peking, Vetch].

Atwood, Christopher P., 1992-1993 The Marvellous Lama in Mongolia: the Phenomenology of a Cultural Borrowing, Acta Orientalia Hungaricae Academiae Scientiarum, 46(1), pp. 3-30.

Atwood, Christopher P., 2000 Worshipping Grace: The Language of Loyalty in Qing China, Late Imperial China 21(2) (December), pp. 86-139.

Aubin, Françoise 1987 A Note about the Spread of Chinese Literature amongst the Mongols, in Claudine Salmon (éd.), Literary Migrations : Traditional Chinese Fiction in Asia (17-20 $0^{\text {th }}$ Centuries) (Pékin, International Culture Publishing).

Aubin, Françoise 2010 To Impress the Seal: A Technological Transfer, in Isabelle Charleux, Roberte Hamayon, Grégory Delaplace et Scott Pearce (éd.), Representing Power in Ancient Inner Asia: Legitimacy, transmission and the sacred (Bellingham: Western Washington University), pp. 159-208.

Baddeley, John Frederick 1919 Russia, Mongolia, China, being some Record of the Relations between them from the beginning of the 17th Century to the Death of Tsar Alexei Mikhailovich A.D. 1602-1676 (Londres, Macmillan), 2 vol.

Bawden, Charles R. (éd.) 1961 The Jebtsundamba Khutukhtus of Urga, texte, trad. et notes critiques d'un ouvrage mongol sur les biographies des Jebcündamba qutuүtu daté de 1859 (Wiesbaden, Otto Harrassowitz) (Asiatische Forschungen, 9).

\footnotetext{
${ }^{66}$ Voir également le roman d'arts martiaux Luding ji 鹿鼎記, écrit par Jin Yong 金庸 (1973), traduit en anglais : The Deer and the Cauldron, plusieurs fois adapté au cinéma et à la télévision, dans lequel Kangxi apparait comme un personnage secondaire.
} 
Bawden, Charles R.

[1968] 1989 The Modern History of Mongolia (Londres \& New York: Kegan Paul International).

Berger, Patricia et Terese Tse Bartholomew (éd.) 1995 Mongolia : the Legacy of Chinggis Khan (Londres/New York, Thames and Hudson; San Francisco (Ca.), Asian Art Museum of San Francisco).

Birtalan, Àgnes 2005 The Mongolian Great Khans in Mongolian Mythology and Folklore, Acta Orientalia Academiae Scientiarum Hung. 58/3, pp. 299-311.

Bouvet, P. Joachim [1699] Histoire de l'empereur de la Chine présentée au Roy (Tientsin, Compagnie de Jésus) [La Haye, Meyndert]

Bredon, Juliette 1922 Peking. An Historical and Intimate Description of its Chief Places of Interest (Shanghai/Hong Kong/Singapour/Hankow/Yokohama, Kelly \& Walsh).

Cerensodnom, D. 1982 Mongol ardyn ülger (Ulaanbaatar, Ulsyn hevlelijn gazar).

Chang, Michael G. 2007 A Court on Horseback : Imperial Touring and the Construction of Qing Rule, 1680-1785 (Cambridge, Mass./London, Harvard University Press).

Charleux, Isabelle 2006 Temples et monastères de Mongolie-Intérieure (Paris, Comité des Travaux Historiques et Scientifiques/Institut National d'Histoire de l'Art) (+ 1 CD rom).

Charleux, Isabelle à paraître Nomads on Pilgrimage. Mongols on Wutaishan (China), 1800-1940 (titre provisoire).

Chiodo, Elisabetta 2011 The Walther Heissig Collection of Mongolian Oral Literature (Paderborn/Münich/Zürich, Verlag Ferdinand Schöningh).

Chou, Wen-shing 2007 Ineffable Paths : Mapping Wutaishan in Qing Dynasty China, The Art Bulletin, 89(1) (mars), pp. 108-129.

Cimeddorji 1991 Die Briefe des K'ang-hsi Kaiser aus den Jahren 1696-97 an den Kronprinzen Yin-ch'eng aus mandschurischen Geheimdokument : ein Beitrag zum ersten Dsungarenkrieg der Ch'ing 1690-1697 (Wiesbaden, Otto Harrassowitz).

Delege 德勒格 [Deleg Delger] 1998 Nei Menggu lamajiao shi 內蒙古喇嘛教史 [Histoire du «lamaïsme» en Mongolie-Intérieure] (Hohhot, Nei Menggu renmin chubanshe). 
Du Halde, Jean-Baptiste (SJ) 1735 Description géographique, historique, chronologique, politique et physique de l'empire de la Chine et de la Tartarie (Paris, P.G. Le Mercier), 4 vol.

Elverskog, Johan 2006 Our Great Qing: The Mongols, Buddhism, and the State in Late Imperial China (Honolulu, University of Hawai'i Press).

« Face Music - Projects - Tsam Dance Masks - Mongolia », P \& C Face Music - February 2007 - Albi. http://www.face-music.ch/tsam/mask_kashmiren.html (consulté le 2 novembre 2011).

Fang Qingqi 方慶奇 et al. 1985 Wutaishan fengwu chuanshuo 五臺山風物五傳說 (Taiyuan, Shanxi renmin chubanshe).

Farquhar, David M. 1978 Emperor as Bodhisattva in the Governance of the Ch'ing Empire, Harvard Journal of Asiatic Studies, 38(1) (juin), pp. 5-34.

Fontein, Jan 1999 The Dancing Demons of Mongolia (Londres, Lund Humphries Publishers/John Vrieze).

Guisui xian zhi 歸綏縣志 [Monographie de Gui(hua) Sui(yuan)] [1934] 1968 de Zheng Yufu 鄭裕孚 et Zheng Zhichang 鄭植昌 (Beijing), 1934. - Éd. Taipei, Chengwen chubanshe, 1968 (Zhongguo fangzhi congshu, Saibei difang, 10).

Halén, Harry 1987 Mirrors of the Void, Buddhist Art in the National Museum of Finland, 63 Sino-Mongolian thangkas from the Wutaishan workshops, a panoramic map of the Wutai Mountains and objects of diverse origins (Helsinki, National Board of Antiquities).

Hamayon, Roberte et Namtcha Bassanoff 1973 De la difficulté d'être une belle-fille, Études mongoles, 4, pp. 7-74.

Hedley, John 1910 Tramps in Dark Mongolia (New York, Scribner's Sons).

Heissig, Walther 1999 Problems Arising from some of Mostaert's Ordos Tales : Additions and Consequences, in Klaus Sagaster (éd.), Antoine Mostaert (1881-1971) - C.I.C.M. Missionary and Scholar. Volume One : Papers (Louvain, Ferdinand Verbiest Foundation/China-Europe Institute), pp. 73-84 (Louvain Chinese Studies IV.)

Huc, Régis Evariste [1924] 1987 Souvenirs d'un voyage dans la Tartarie et le Thibet pendant les années 1844, 1845 et 1846, éd. revue et commentée par le père J. M. Planchet (Paris, L'Astrolabe-Peuples du monde) [Pékin], 2 vol. 
Hummel, Arthur W. (éd.) 1943-1944 Eminent Chinese of the Ch'ing Period (1644-1912) (Washington, The Library of Congress, 2 vol.)

Humphrey, Caroline 2007 Vital Force : the Story of Dugar Jaisang and Popular Views of Mongolian-Tibetan Relations from Mongolian Perspectives, in Uradyn E. Bulag et Hildegard G.M. Diemberger (éd.), The Mongolia-Tibet Interface : Opening New Research Terrains in Inner Asia. Proceedings of the Tenth Seminar of the IATS, 2003 (Leyde/Boston, Brill), pp. 159-174 (Brill's Tibetan Studies Library, vol. 9).

Humphrey, Caroline 2010 The Ruler and the lama : political and religious relations in the Urad West Duke Banner in the eighteenth and nineteenth centuries, in Isabelle Charleux, Roberte Hamayon, Grégory Delaplace et Scott Pearce (éd.), Representing Power in Modern Inner Asia: Conventions, alternatives and oppositions (Bellingham, Western Washington University), pp. 183-219.

Jin Yong 金庸 1973 Luding ji 鹿鼎記 (Hong Kong: Wugong Press). Traduit en anglais par John Minford 1997 The Deer and the Cauldron: the Adventures of a Chinese Trickster (Oxford : Oxford University Press).

Kangxi nanxun miji 康熙南巡秘記 1916 [1701?] s. n. (Shanghai: Zhonghua).

Köhle, Nathalie 2008 Why Did the Kangxi Emperor Go to Wutai Shan ?: Patronage, Pilgrimage, and the Place of Tibetan Buddhism at the Early Qing Court, Late Imperial China, 29(1), pp. 73-119.

Li Dezhu 李德洙 et al. 1994 Zhongguo shaoshu minzu wenhua shi 中國少數民族文化史 (Shenyang, Liaoning renmin chubanshe).

Li Shujiang et Karl W. Luckert 1994 Mythology and Folklore of the Hui (Albany, State University of New York Press).

Lovell, Julia 2006 The Great Wall : China Against the World, 1000 BC - AD 2000 (New York, Grove Press).

Lu Minghui 盧明輝 1987 Bailing miao 百靈廟 [Le Beile-yin süme], in Nei Menggu wenwu guji sanji 內蒙古文物古跡散集 (Hohhot, Nei Menggu renmin chubanshe), pp. 162-168.

Majer, Zsuzsa 2008 A Comparative Study of the Ceremonial Practice in Present-day Mongolian Monasteries, PhD. Dissertation (Eötvös Loránd University, Department of Inner Asian Studies, Budapest). 
Markham, Clements R. (dir.) 1876 Narrative of the Mission of George Bogle to Tibet, and of the Journey of Thomas Manning to Lhāsa, with notes, etc. (Londres, Trübner \& co), $2^{\mathrm{e}}$ éd.

Narantuya, C. 2005 Mongol urlagiin ünet büteelü̈̈. Ayuurzanyn Altangereliin cugluulgaac / Treasures of Mongolian Art. Collection of Altangerel Ayurzana (Ulaanbaatar, The Asian Art antique gallery).

Pegg, Carole 2001 Mongolian Music, Dance and Oral Narrative. Performing Diverse Identities (Seattle/London, University of Washington Press).

Perdue, Peter C. 2005 China Marches West: the Qing Conquest of Central Eurasia (Cambridge, Harvard University Press).

Pozdneev, Aleksej M. [1896-1898] 1977 Mongolia and the Mongols, vol. II, trad. du russe par W. Dougherty (Bloomington, Mouton \& Co.) [Saint-Pétersbourg].

Prjevalsky, Lieut.-Colonel Nicholaj [1875] 1880 Mongolie et pays des Tangoutes, trad. du russe par G. du Laurens (Paris, Hachette).

Qianlong xia Jiangnan 乾隆下江南 [XIX ${ }^{\mathrm{e}}$ siècle ?] 1997 s.n., XIX ${ }^{\mathrm{e}}$ siècle - Éd. Zhongzhou guji chubanshe, 1997.

Qiao Ji 喬吉 (=Coiji) $1994 \mathrm{Nei}$ Menggu simiao 內蒙古寺廟 [Les monastères de Mongolie-Intérieure] (Hohhot, Nei Menggu renmin chubanshe) (Nei Menggu lishi wenhua congshu).

Qinding Qingliangshan zhi 欽定淸凉山志 [Monographie locale impériale du Qinglianshan]

compilée en 1785, imprimée par la Maison Impériale du Palais en 1811, 22 chap.

Qingbai leichao 清稗類鈔 [Anecdotes classifiées des Qing] [1917] 1984-1986 Compilé par Xu Ke 徐珂 (1869-1928), 1917 - Éd. Pékin, Zhonghua shuju, 1984-1986.

Ripa, M. 1844 Memoirs of Father Ripa during Thirteen Year's Residence at the Court of Peking in the Service of the Emperor of China, sélectionné et traduit de l'italien par Fortunato Prandi (Londres, John Murray).

Sagaster, Klaus 1967 Subud Erike, ein Rosenkranz aus Perle, Die Biographie des 1. Pekinger 1Can skya Khutukhtu Nag dban blo bzan c'os ldan, verfasst von Nag dban c'os ldan alias ses rab das rgyas (Wiesbaden, Otto Harrassowitz). 
Sai Wendusu 塞·文都素 $1961 \mathrm{Gu}$ hua “Yueming lou” jianjie 古畫“月明樓” 簡介, Wenwu 文物, 1961-9, p. 63.

Schrempf, Mona 2006 Hwa shang at the Border: Transformations of History and Reconstructions of Identity in Modern A mdo, Journal of the International Association of Tibetan Studies 2 (August), pp. 1-32, http://www.thlib.org?tid=2721 (consulté le 1er avril 2011).

Spence, Jonathan D. 1974 Emperor of China: Self-Portrait of K'ang-hsi (London, Jonathan Cape).

Spence, Jonathan D. 1966 Ts'ao Yin and the K'ang-hsi Emperor : bondservant and master (New Haven, Yale University Press) (Yale historical publications, 85)

Temür, Altan, Sonum et B. Caүankeüken 2009 Qara ükertü kübegün-ü üliger (Hohhot, Ündüsüten-ü keblel-ün qorija).

Veit, Veronika 1982 Galdan's nephew Danjila (d. 1708) : An example of the K'ang-hsi emperor's successful policy towards the Mongols, Asian and African Studies, 16, pp. 345356.

Waddel, L. Austine [1935] 1939 The Buddhism of Tibet or Lamaism (Cambridge, W. Heffers \& Sons).

Shengwu ji 聖武記 [《S Sainte puissance militaire », Histoire des campagnes impériales] 1842 de Wei Yuan 魏源 (1794-1857), 14 chap. - Éd. Shanghai : Zhonghua shuju, 1936.

Wei Guozuo 魏国祚 [1988] 1993 Wutaishan daoyou 五臺山导游 (Beijing, Zhongguo lüyou chubanshe), $2^{\mathrm{e}}$ ed.

Weiers, Michael 2000 Die politische Dimension des Jadesiegels zur Zeit des Mandschuherrschers Hongtaiji, Zentralasiastische Studien, 30, pp. 103-124.

Xiaoting xulu 嘯亭續錄 [XVIII ${ }^{\mathrm{e}}$ siècle] 1996 de Zhaolian 昭槤 (1780-1833), 3 juan. - Éd. Xuxiu siku quanshu, (Shanghai, Shanghai guji chubanshe), 1996.

Yang Junlei 2008 Auspicious clouds above the Mountain Wutai, traduit du chinois par Gaohua Linhui (Taiyuan, Shanxi kexue chubanshe).

Yang Zengwu 楊增武 2005 Huangjia yu Wutaishan 皇家與五臺山 (Taiyuan, Shanxi guji chubanshe). 
Zhang Yuxin 張羽新 1988 Qing zhengfu yu lamajiao 清政府與喇嘛教 (Lhasa, Xizang renmin chubanshe).

Zhao Chunping 趙春萍 et al. 1987 Nei Menggu lüyou 內蒙古旅游 [Guide de tourisme en Mongolie-Intérieure] (Hohhot, Nei Menggu renmin chubanshe).

\section{Illustrations}

Fig. 1. Hašin han et ses fils au festival 'cham d'Ulaanbaatar, avant 1937. Fontein 1999, fig. 15 , p. 51

Fig. 2. Zanabazar donnant une initiation à l'empereur Kangxi et à la " mère de la nation »

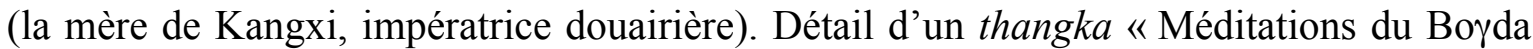
gegen », fin XIX ${ }^{\mathrm{e}}$-début du XX $\mathrm{X}^{\mathrm{e}}$ siècle, 265,7x151,4 cm, Musée du Palais du Bogd Han, Ulaanbaatar. Berger et Tse Bartholomew (éd.) 1995, 130

Fig. 3. La source de jade, devant le Jeke zuu de Hohhot. (C) Isabelle Charleux, 2006.

Fig. 4. Kangxi fait jaillir la source de jade, peinture à Hohhot. Li Dezhu et al. 1994, p.161.

Fig. 5. Kangxi tue le tigre au Wutaishan, carte du Cifusi, 1846, Rubin Museum of Art, New York. (C) Rubin Museum. Karl Debreczeny

Fig. 6. L'arhat Dharmatrāta, sanctuaire du temple de Maitreya (Majidari-jin zuu) à l'est de Hohhot, Mongolie-Intérieure. (C) Gilles Béguin

Fig. 7. Copie récente de la peinture «Yueminglou », 3,5 $\mathrm{m}$ de long sur 1,3 $\mathrm{m}$ de large, située dans le Jeke zuu de Hohhot, 2003. L'original, daté des années 1889-1893, est conservée dans le musée d'Histoire de Hohhot. (C) Joseph Bonnemaire

Fig. 8. Série télévisée 《Kangxi weifu sifangji»康熙微服私訪記 (《Les voyages incognito de Kangxi »). 